\title{
Episodic construction of the Tatra granitoid intrusion (Central Western Carpathians, Poland/Slovakia): consequences for the geodynamics of Variscan collision and Rheic Ocean closure
}

\author{
Aleksandra Gawęda ${ }^{1}$ Jolanta Burda ${ }^{1}$ - Urs Klötzli ${ }^{2}$ Jan Golonka ${ }^{3}$. \\ Krzysztof Szopa ${ }^{1}$
}

Received: 24 March 2015 / Accepted: 8 August 2015 / Published online: 21 August 2015

(C) The Author(s) 2015. This article is published with open access at Springerlink.com

\begin{abstract}
The Tatra granitoid pluton (Central Western Carpathians, Poland/Slovakia) is an example of composite polygenetic intrusion, comprising many magmatic pulses varying compositionally from diorite to granite. The $\mathrm{U}-$ $\mathrm{Pb}$ LA-MC-ICP-MS zircon dating of successive magma batches indicates the presence of magmatic episodes at 370$368,365,360,355$ and 350-340 Ma, all together covering a time span of $30 \mathrm{Ma}$ of magmatic activity. The partial resorption and recycling of former granitoid material ("petrological cannibalism") was a result of the incremental growth of the pluton and temperature in the range of $750-850{ }^{\circ} \mathrm{C}$. The long-lasting granitoid magmatism was connected to the prolonged subduction of oceanic crust and collision of the Proto-Carpathian Terrane with a volcanic arc and finally with Laurussia, closing the Rheic Ocean. The differences in granitoid composition are the results of different depths of crustal melting. More felsic magmas were generated in the outer zone of the volcanic arc, whilst more mafic magmas were formed in the inner part of the supra-subduction zone. The source rocks of the granitoid magmas covered the compositional range of metapelite-amphibolite and were from both lower and upper crust. The presence of the inherited zircon cores suggests that the collision and granitoid magmatism involved crust of Cadomian consolidation age (c. 530 and $518 \mathrm{Ma}$ ) forming the Proto-Carpathian Terrane,
\end{abstract}

Aleksandra Gawęda

aleksandra.gaweda@us.edu.pl

1 Faculty of Earth Sciences, University of Silesia, ul. Bedzińska 60, 41-200 Sosnowiec, Poland

2 Department of Lithospheric Research, University of Vienna, Althanstrasse 14, 1090 Vienna, Austria

3 Faculty of Geology, Geophysics and Geotourism, Mickiewicza 30 St., Kraków, Poland crust of Avalonian affinity (462, $426 \mathrm{Ma}$ ) and melted metasedimentary rocks of volcanic arc provenance.

Keywords Granitoid · Magmatic pulses - Variscan orogeny $\cdot$ Proto-Carpathian Terrane

\section{Introduction}

The formation of many granitoid intrusions is considered to result from the incremental assembly of felsic magmas over different periods of time (e.g. Coleman et al. 2004; Glazner et al. 2004; Paterson et al. 2011), usually with addition of mafic components (Słaby and Martin 2008). The most efficient natural migration channels for magmas of different compositions are shear zones, enabling a continuous supply of magma (Petford et al. 2000; Pawley et al. 2002; Liotta et al. 2008; Oberc-Dziedzic et al. 2013). In the case of large-scale, long-lived shear zones, transporting melts and crystal-melt mushes, plutons can be fed by partial melting of both crustal and mantle sources, in different proportions. The interaction of melts from different sources produces granitoids of mixed geochemical and isotopic signatures.

The formation of the European Variscides has been interpreted as a result of the collision of microterranes, rifted away from the Gondwana margin and docked onto Laurussia (Nance et al. 2010; Gawęda and Golonka 2011). The resulting Upper Devonian-Carboniferous granitoid magmatism and metamorphism, and the tectonic zonation have been described in detail from many Western European localities. The core mountains in the Carpathian orogenic belt, being fragments of the Variscan continental crust, were subsequently incorporated into the Alpine units (i.e. Tatricum, Veporicum, Gemericum, Zemplinicum; Ebner et al. 2008). At present they bring significant information 
Fig. 1 A simplified geological sketch of the Carpathian Chain with geographic co-ordinates with the location of the Central Western Carpathians (a), the position of the Variscan crystalline cores in the Central Western Carpathians with the marked location of the Tatra Mountains (b) and schematic geological map of the Tatra Mountains with the location of samples used for zircon $\mathrm{U}-\mathrm{Pb}$ dating (c). I Sub-Tatric Fault, II Ružbachy Fault, III Choč Fault, $I V$ Krowiarki Fault

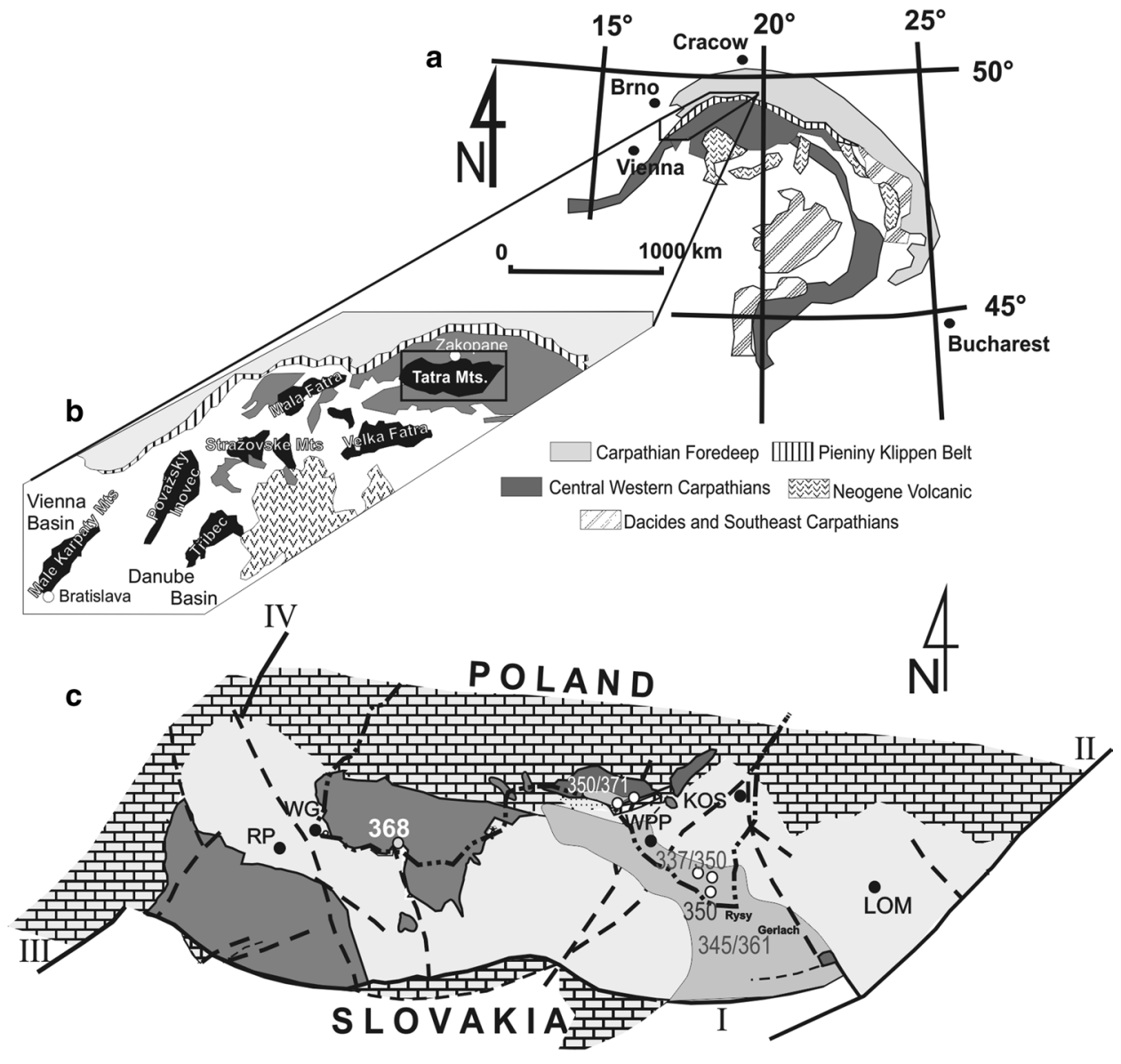

metamorphic rocks $\square$ granitoids (Cycle 1) $\square$ granitoids (Cycle 2) 臣 sedimentary cover faults (identified and inferred)

- sampling points $\mathrm{O}$ samples dated by zircon U-Pb method on the subduction- and collision-related voluminous granitoid intrusions and metamorphism, dated to the same Upper Devonian-Carboniferous period (e.g. Broska et al. 2013).

In this study we deal with the Tatra granitoid pluton, which is a key point for understanding the Variscan geological history of the Inner Western Carpathians (Fig. 1a). The Tatra Massif is the northernmost crystalline massif in the Inner Western Carpathians (Fig. 1a), weakly affected by Alpine deformation (Jurewicz 2005) and offers a unique opportunity to trace the pre-Alpine geological processes. The Tatra granite is a synkinematic, polygenetic tongueshaped pluton with well-developed magmatic layering and associated cumulates of unusual composition, genetically connected to the mixing-mingling of felsic and mafic magmas, all located in the same syntectonic intrusion, showing the same flow direction and formed by multiple magma injection (Kohut and Janak 1994; Gawęda 2008; Gawęda and Szopa 2011; Burda et al. 2011; Szopa et al. 2013). This provides an excellent opportunity to study the time relationships of subduction-related magmatic activity in the shear zone, the development of the volcanic arc and the final Variscan collision of Laurussia with Gondwana.

The aim of this paper is to investigate the zircon $\mathrm{U}-\mathrm{Pb}$ ages of the successive episodes of granitoid magma intrusion, including the problem of rejuvenation of older magmatic material during subsequent magmatic pulses. The consequences for the plate tectonic interpretation of the Carpathians realm are also discussed.

\section{Geological setting}

The Tatra Mountains block represents one of the core mountains in the Central Western Carpathians (Fig. 1a-c). The core mountains are uplifted portions of the Variscan crust tectonically emplaced among Alpine structures of the Carpathian mountain chain. In the Tatra Mountains a crystalline, pre-Alpine core is overlain by Mesozoic sedimentary formations. The crystalline core is composed of a composite granitoid intrusion and its metamorphic envelope. 
Metamorphic envelope rocks are preserved mostly in the western part of the massif, termed the Western Tatra Mountains (Fig. 1c), and were intensively migmatised at ca. 358-365 Ma (Burda and Gaweda 2009). The layered Tatra pluton (Gawęda and Szopa 2011) comprises several magmatic pulses: (1) I-type mingled hybrid quartz diorites, interpreted as mafic precursors (Gawęda et al. 2005), (2) granodiorie-tonalite, called "common Tatra type" (Kohut and Janak 1994) or Koszysta-type (Morozewicz 1914) and (3) syenogranite-monzogranite, locally porphyritic, rich in xenoliths and enclaves (Gawęda 2008, 2009), called "High Tatra type" (Fig. 1c). Available zircon U-Pb data define the range of granitoid intrusion for the interval as: $368 \pm 8 \mathrm{Ma}$ (for mafic precursors; Burda et al. 2011), $363 \pm 11-347 \pm 14 \mathrm{Ma}$ (granites of common Tatra type; Poller et al. 2000) and 350 $\pm 5-337 \pm 6 \mathrm{Ma}$ (granites of High Tatra type; Burda et al. 2013a). The historical term of "Goryczkowa-type" granites, used in contradiction to "Koszysta-type" granites (Morozewicz 1914; Kohut and Janak 1994), was thought to be restricted to crystalline portions, unrooted, forming the cores of Alpine nappes, but recent investigations have pointed to the petrological and geochronological similarities of these granitoids, dated at $371 \pm 6 \mathrm{Ma}$ to the "common Tatra type" granites and their mafic precursors (Burda et al. 2011, 2013b). The dextral top-to-SE direction of tabular pluton emplacement matches the stress field of the migmatised metamorphic envelope, suggesting a syntectonic character of the intrusion (Kohut and Janak 1994; Gawęda and Szopa 2011). Variscan exhumation of the Tatra crystalline massif at ca. $340 \mathrm{Ma}$ with an approximate $30 \%$ m.y. cooling rate (Moussallam et al. 2012) post-dated granite intrusion which is consistent with $\mathrm{U}-\mathrm{Pb}$ apatite cooling ages of ca $340 \mathrm{Ma}$ (Gawęda et al. 2014).

Late Cretaceous-Early Cainozoic folding and thrusting, linked to the closure of the Tethys Ocean (Golonka et al. 2003), dislocated the Tatra Massif to the present position as a part of the Central Western Carpathians (Gawęda and Golonka 2011). Paleogene uplift of the whole Tatra Massif was associated with its division into small tectonic blocks (36-24 and 10 Ma; Kohut and Sherlock 2003; Burchart 1972). During the Quaternary, the entire massif was tilted to the north along the currently active sub-Tatric Fault (IFig. 1c). The present-day tilting angle is deemed to be ca $30^{\circ}$ (Grabowski and Gawęda 1999).
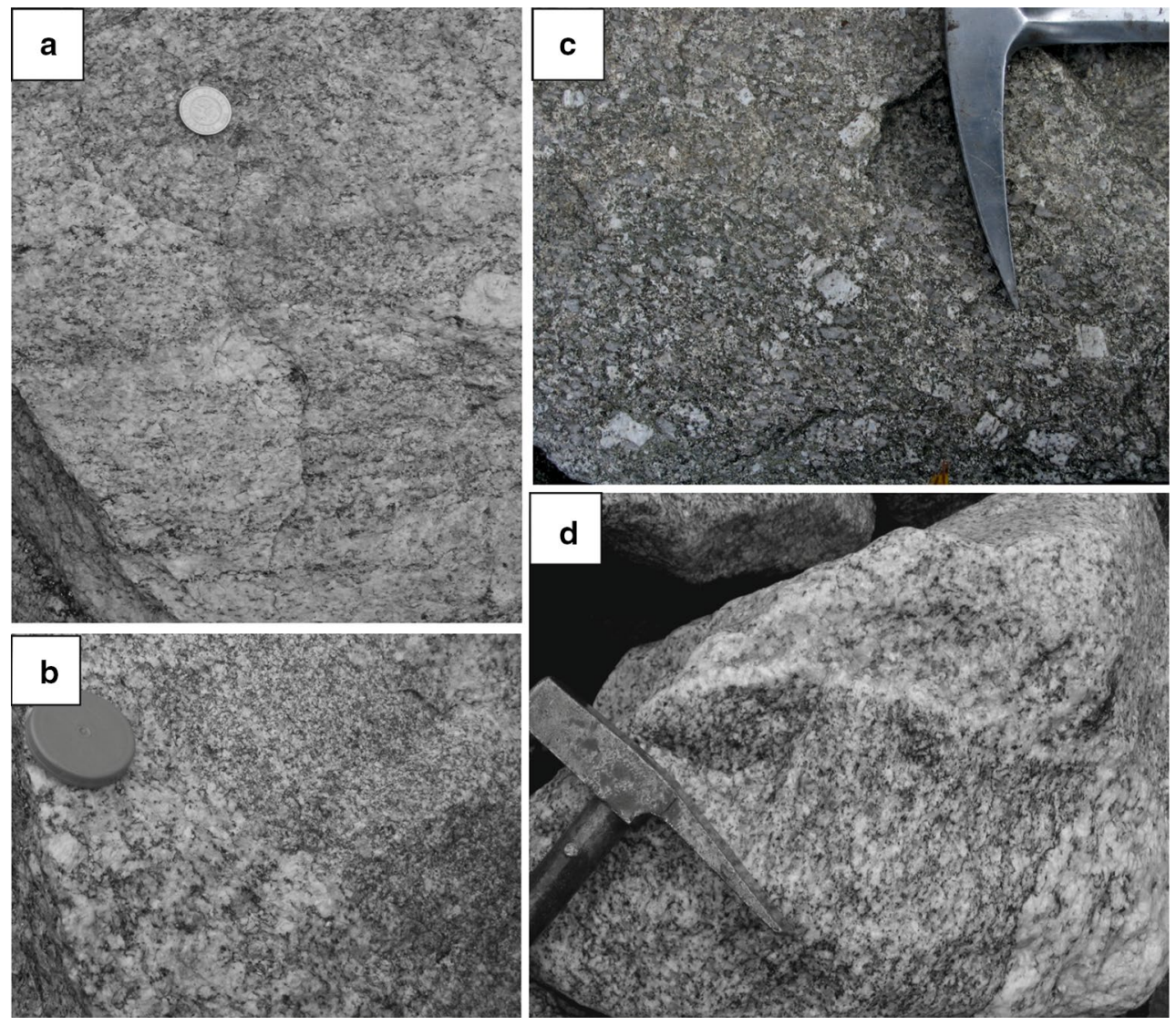

Fig. 2 Textures of the Tatra granite: a layered granitoid, b homogeneous granitoid with K-feldspar porphyrocrysts, c sharp contact between two types of granite: equigranular biotite monzogranite (upper) and porphyritic syenogranite (lower), $\mathbf{d}$ enclave of older granodiorite in the more felsic younger granite 
Table 1 Representative microprobe analyses of magmatic micas and their crystal-chemical formulae, recalculated to $22 \mathrm{O}^{2-}$

\begin{tabular}{|c|c|c|c|c|c|c|c|c|}
\hline \multirow{2}{*}{$\begin{array}{l}\text { Mineral } \\
\text { Component }\end{array}$} & \multicolumn{6}{|l|}{ Biotite } & \multicolumn{2}{|l|}{ Muscovite } \\
\hline & Bt1-KOS & $\mathrm{Bt} 2-\mathrm{KOS}$ & Bt3-WG & Bt4-RP & Bt5-LOM & Bt1-WPP & Ms1-KOS & Ms2-KOS \\
\hline $\mathrm{SiO}_{2}$ & 35.58 & 36.75 & 35.81 & 35.99 & 36.00 & 34.87 & 45.60 & 45.29 \\
\hline $\mathrm{TiO}_{2}$ & 3.35 & 3.27 & 3.26 & 3.43 & 2.04 & 3.58 & 1.31 & 1.35 \\
\hline $\mathrm{Al}_{2} \mathrm{O}_{3}$ & 17.51 & 16.57 & 17.43 & 16.44 & 17.50 & 16.97 & 32.11 & 32.46 \\
\hline $\mathrm{Cr}_{2} \mathrm{O}_{3}$ & 0.03 & 0.05 & 0.03 & 0.00 & 0.00 & 0.08 & 0.02 & 0.00 \\
\hline $\mathrm{FeO}$ & 18.85 & 17.77 & 21.28 & 19.14 & 20.25 & 22.58 & 4.38 & 4.20 \\
\hline $\mathrm{MgO}$ & 9.69 & 11.43 & 8.36 & 10.28 & 9.60 & 0.35 & 0.80 & 0.81 \\
\hline $\mathrm{MnO}$ & 0.46 & 0.42 & 0.37 & 0.30 & 0.52 & 7.56 & 0.00 & 0.00 \\
\hline $\mathrm{Na}_{2} \mathrm{O}$ & 0.10 & 0.12 & 0.12 & 0.11 & 0.15 & 0.13 & 0.38 & 0.40 \\
\hline $\mathrm{K}_{2} \mathrm{O}$ & 9.82 & 9.57 & 9.68 & 9.39 & 10.00 & 9.67 & 10.93 & 10.89 \\
\hline $\mathrm{BaO}$ & 0.27 & 0.21 & 0.00 & 0.28 & 0.05 & 0.39 & 0.00 & 0.00 \\
\hline Total & 95.66 & 96.16 & 96.34 & 95.34 & 96.15 & 96.18 & 95.53 & 95.40 \\
\hline \multicolumn{9}{|c|}{ Crystal-chemical formulae recalculated for $22 \mathrm{O}^{2-}$} \\
\hline $\mathrm{Si}$ & 5.421 & 5.525 & 5.462 & 5.499 & 5.489 & 5.394 & 6.185 & 6.148 \\
\hline $\mathrm{Al}^{\mathrm{IV}}$ & 2.579 & 2.475 & 2.538 & 2.501 & 2.511 & 2.606 & 1.815 & 1.852 \\
\hline $\mathrm{Al}^{\mathrm{VI}}$ & 0.566 & 0.461 & 0.595 & 0.459 & 0.635 & 0.487 & 3.318 & 3.340 \\
\hline $\mathrm{Ti}$ & 0.385 & 0.370 & 0.374 & 0.394 & 0.233 & 0.417 & 0.133 & 0.138 \\
\hline $\mathrm{Cr}$ & 0.003 & 0.006 & 0.004 & 0.000 & 0.000 & 0.010 & 0.002 & 0.000 \\
\hline $\mathrm{Fe}$ & 2.402 & 2.234 & 2.714 & 2.446 & 2.582 & 2.921 & 0.497 & 0.477 \\
\hline $\mathrm{Mg}$ & 2.201 & 2.562 & 1.902 & 2.341 & 2.182 & 0.046 & 0.162 & 0.163 \\
\hline $\mathrm{Mn}$ & 0.059 & 0.053 & 0.048 & 0.038 & 0.067 & 1.743 & 0.000 & 0.000 \\
\hline $\mathrm{Na}$ & 0.028 & 0.036 & 0.034 & 0.033 & 0.044 & 0.039 & 0.101 & 0.106 \\
\hline $\mathrm{K}$ & 1.910 & 1.836 & 1.883 & 1.830 & 1.946 & 1.908 & 1.891 & 1.885 \\
\hline $\mathrm{Ba}$ & 0.016 & 0.012 & 0.000 & 0.017 & 0.003 & 0.024 & 0.000 & 0.000 \\
\hline$\# f m$ & 0.530 & 0.472 & 0.579 & 0.515 & 0.587 & 0.630 & 0.754 & 0.745 \\
\hline
\end{tabular}

\section{A brief outline of the Palaeozoic geodynamics of the Central Western Carpathian area}

The Palaeozoic history of the circum-Carpathian realm began with the accretion of Avalonia to Baltica during the Silurian closure of the Tornquist Sea and formation of Laurussia. This accretion was followed by the development of the subduction zone, dipping towards Laurussia and marked by Andean-type igneous activity at 450-420 Ma. In the Central Western Carpathians that episode is represented by $470-435 \mathrm{Ma}$ tonalitic orthogneisses and metagabbros in the Veporic and Tatric units (Putiš et al. 2008; Janák et al. 2002; Gaab et al. 2003). As a result of the slab roll-back, rifting developed at the southern margin of Laurussia, initiating the formation of a new oceanic basin and the derivation of the ribbon-like microplates. These ribbon-like microplates probably included the Proto-Carpathian Terrane (Gawęda and Golonka 2011; Golonka and Gawęda 2012 and references therein). The occurence of Early Devonian ophiolites recognised in the Tatric and Gemeric units of the Carpathians and corresponding to the Lizard and Central Sudetic ophiolite is the record of the oceanic crust (Ebner et al. 2008). The Early Devonian ages of the oceanic crust in the Central Western Carpathians (371 Ma for the Tatricum unit and 383-385 Ma for the Gemericum unit; Putiš et al. 2009) and formation of the Lahn-Dill volcano-sedimentary complexes (394 Ma; Kohut et al. 2006) indicate the association of the Proto-Carpathian Terrane rather with the Rheno-Hercynian back-arc basin than with the main branch of the Rheic Ocean (Gawęda and Golonka 2011; Golonka and Gawęda 2012).

\section{Sampling and analytical methods}

Geological observations were made, and sampling was done over the whole of the Tatra intrusion (Fig. 1c). The selected vertical profiles were sampled in detail for petrographical and geochemical investigations. Representative samples of granitoids, weighing from 15 to $25 \mathrm{~kg}$, were collected for zircon dating with permission of the Polish Ministry of Environment and the Tatra National Park. 
Fig. 3 Microtextures of the Tatra granite: a K-feldspar with rows of plagioclase inclusions and antecrystic unzoned core, b disoriented inclusions of plagioclase, biotite and quartz in alkali feldspar, $\mathbf{c}$ broken and displaced zoned plagioclase crystal, sealed by the finegrained quartz-alkali feldspar matrix, $\mathbf{d}$ ductile deformed plagioclase, e two-stage growth of K-feldspar, documented by the compositional profile (f), showing changes in celsjan $(\mathrm{Cn})$ molecule, $\mathrm{g}$ partially resorbed xenocryst of quartz with biotite inclusions inside the monzogranite enclave
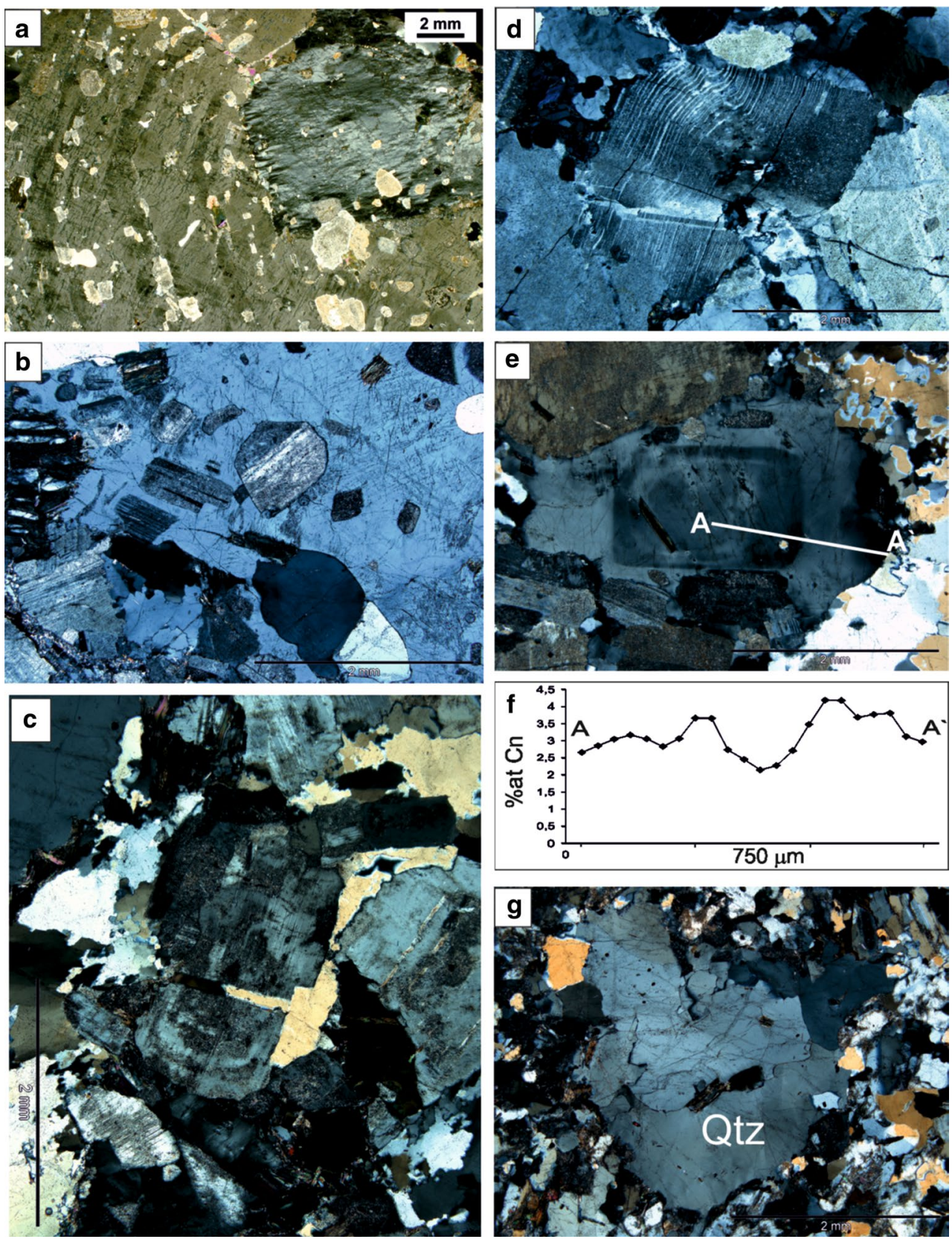

\section{Microscopy and whole-rock analysis}

Microscopic observations were carried out at the Faculty of Earth Sciences, University of Silesia, using an Olympus BX-51 microscope, in order to select representative samples for whole-rock geochemical and isotopic investigations and for $\mathrm{U}-\mathrm{Pb}$ zircon dating. Samples weakly affected by secondary alteration (see Gawęda and Włodyka 2012) were selected for whole-rock analyses. The latter were done by XRF for major and large ion lithophile trace elements (LILE) and by ICP-MS for high field strength elements (HFSE) and rare earth elements (REE) in the ACME Analytical Laboratories (Canada) according to procedures described on http://acmelab.com. Preparation involved lithium borate fusion and dilute digestions or hot four-acid digestion for ICP-MS, $\mathrm{LiBO}_{2}$ fusion for XRF and lithium borate decomposition or aqua regia digestion for ICP-MS. Lost of ignition (LOI) was determined at $1000{ }^{\circ} \mathrm{C}$. REE were normalised to $\mathrm{C} 1$ chondrite (Sun and McDonough 1989).

\section{U-Pb zircon dating}

Seven granitoid samples were selected for U-Pb zircon dating. Zircon crystals were separated using standard techniques (crushing, hydrofracturing, washing, Wilfley table, 
Table 2 Chemical composition of amphibole crystals and parent melt parameters calculated according to procedures of Ridolfi et al. (2010)

\begin{tabular}{|c|c|c|c|}
\hline Component (wt\%) & Amph 1 & Amph 2 & Amph3 \\
\hline $\mathrm{SiO}_{2}$ & 44.54 & 43.80 & 44.60 \\
\hline $\mathrm{TiO}_{2}$ & 0.83 & 0.80 & 0.73 \\
\hline $\mathrm{Al}_{2} \mathrm{O}_{3}$ & 10.16 & 10.59 & 10.01 \\
\hline $\mathrm{Cr}_{2} \mathrm{O}_{3}$ & 0.02 & 0.00 & 0.03 \\
\hline $\mathrm{FeO}$ & 15.80 & 16.46 & 16.05 \\
\hline $\mathrm{MnO}$ & 0.63 & 0.63 & 0.69 \\
\hline $\mathrm{MgO}$ & 11.55 & 11.13 & 11.49 \\
\hline $\mathrm{CaO}$ & 11.83 & 11.94 & 11.70 \\
\hline $\mathrm{Na}_{2} \mathrm{O}$ & 1.17 & 1.12 & 1.17 \\
\hline $\mathrm{K}_{2} \mathrm{O}$ & 0.70 & 0.78 & 0.66 \\
\hline $\mathrm{F}$ & 0.01 & 0.01 & 0.01 \\
\hline $\mathrm{Cl}$ & 0.01 & 0.01 & 0.01 \\
\hline $\mathrm{H}_{2} \mathrm{O}$ (calc) & 1.88 & 1.87 & 1.88 \\
\hline $\mathrm{Fe}_{2} \mathrm{O}_{3}$ (calc) & 6.32 & 6.64 & 6.98 \\
\hline $\mathrm{FeO}$ & 10.12 & 10.49 & 9.78 \\
\hline $\mathrm{O}=\mathrm{F}, \mathrm{Cl}$ & -0.01 & -0.01 & -0.01 \\
\hline Total & 99.77 & 99.80 & 99.72 \\
\hline \multicolumn{4}{|c|}{ Crystal-chemical formulae recalculated to $23 \mathrm{O}^{2-}$} \\
\hline $\mathrm{Si}$ & 6.571 & 6.64 & 6.582 \\
\hline $\mathrm{Al}^{\mathrm{IV}}$ & 1.429 & 10.49 & 1.418 \\
\hline $\mathrm{Al}^{\mathrm{VI}}$ & 0.339 & 0.337 & 0.324 \\
\hline $\mathrm{Ti}$ & 0.092 & 0.089 & 0.081 \\
\hline $\mathrm{Cr}$ & 0.000 & 0.000 & 0.000 \\
\hline $\mathrm{Fe}^{3+}$ & 0.700 & 0.740 & 0.773 \\
\hline $\mathrm{Fe}^{2+}$ & 1.250 & 1.299 & 1.208 \\
\hline Mn & 0.079 & 0.079 & 0.086 \\
\hline $\mathrm{Mg}$ & 2.541 & 2.456 & 2.527 \\
\hline $\mathrm{Ca}$ & 1.871 & 1.894 & 1.850 \\
\hline $\mathrm{Na}$ & 0.334 & 0.321 & 0.335 \\
\hline $\mathrm{K}$ & 0.131 & 0.148 & 0.124 \\
\hline$(\mathrm{Na}+\mathrm{K})(\mathrm{A})$ & 0.336 & 0.363 & 0.308 \\
\hline $\mathrm{Mg} /\left(\mathrm{Mg}+\mathrm{Fe}^{2+}\right)$ & 0.670 & 0.654 & 0.677 \\
\hline $\mathrm{Fe}^{3+} /\left(\mathrm{Fe}^{3+}+\mathrm{Al}^{\mathrm{VI}}\right)$ & 0.674 & 0.687 & 0.704 \\
\hline Species & $\mathrm{Mg}-\mathrm{Hbl}$ & Tschermakitic-Hbl & $\mathrm{Mg}-\mathrm{Hbl}$ \\
\hline$T\left({ }^{\circ} \mathrm{C}\right)$ & 867 & 880 & 860 \\
\hline Uncertainty $\left(\sigma_{\text {est }}\right)$ & 22 & 22 & 22 \\
\hline$P(S)[\mathrm{kbar}]$ & 5.4 & 5.8 & 5.3 \\
\hline$\Delta \mathrm{NNO}$ & 0.70 & 0.6 & 0.7 \\
\hline $\log f \mathrm{O}_{2}$ & -11.80 & -11.60 & -11.9 \\
\hline Uncertainty $\left(\sigma_{\text {est }}\right)$ & 0.4 & 0.4 & 0.4 \\
\hline $\mathrm{H}_{2} \mathrm{O}_{\text {melt }}(\mathrm{wt} \%)$ & 7.50 & 7.60 & 7.5 \\
\hline
\end{tabular}

$P(S)$ pressure calibrated according to Schmidt (1992) procedure magnetic separator and handpicking). The separation was carried out in the Institute of Geological Sciences, Polish Academy of Sciences, Cracow. Zircon grains were selected for morphological study using scanning electron microscopy and then imaged by cathodoluminescence using a FET Philips 30 electron microscope $(15 \mathrm{kV}$ and $1 \mathrm{nA})$ at the Faculty of Earth Sciences, University of Silesia, Sosnowiec, Poland.

Zircon crystals from granitoid rocks were analysed in the Geochronology Laboratory, Institute of Geology at the University of Vienna. Zircon ${ }^{206} \mathrm{~Pb} /{ }^{238} \mathrm{U}$ and ${ }^{207} \mathrm{~Pb} /{ }^{206} \mathrm{~Pb}$ ages were determined using a 193-nm solid state Nd-YAG 

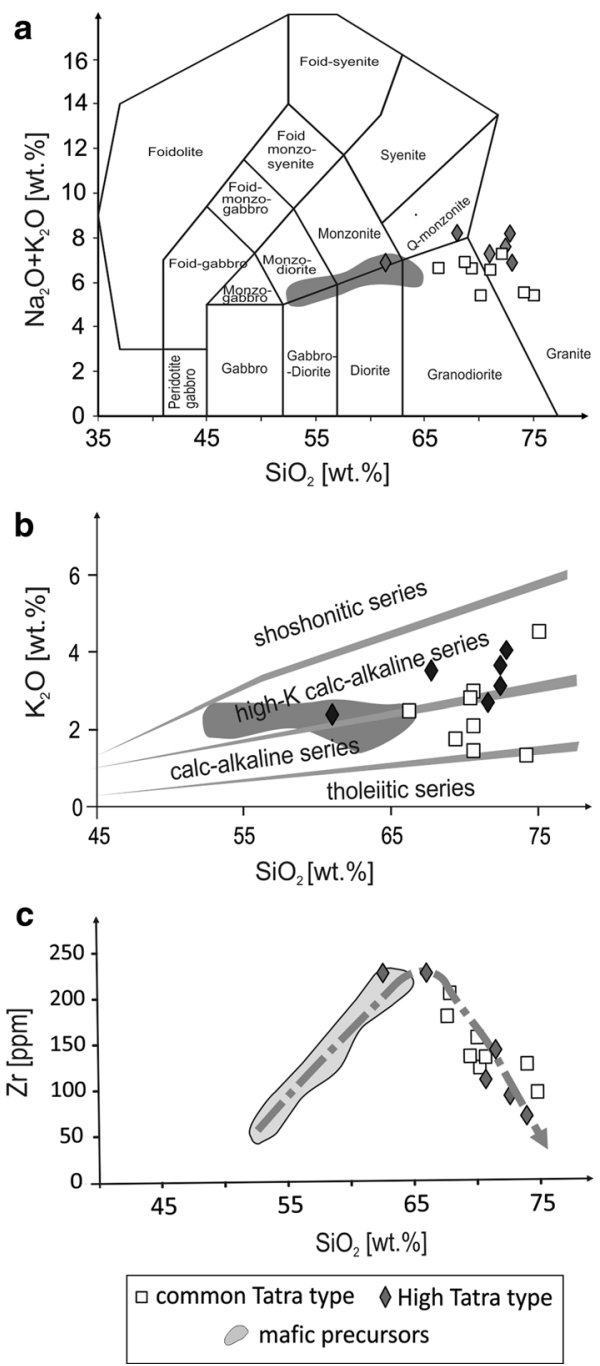

Fig. 4 Classification position of the Tatra Mountains granitoids in: a alkali $\left(\mathrm{K}_{2} \mathrm{O}+\mathrm{Na}_{2} \mathrm{O}\right)$ versus $\mathrm{SiO}_{2}$ TAS classification diagram after Middlemost (1985), b $\mathrm{K}_{2} \mathrm{O}$ versus $\mathrm{SiO}_{2}$ classification diagram after Pecerillo and Taylor (1976), c $\mathrm{Zr}$ versus $\mathrm{SiO}_{2}$ plot. The position of mafic precursors (Gawęda et al. 2005; grey area) is plotted for reference. White squares common Tatra granites, grey diamonds High Tatra granites

laser (NewWave UP193-SS) coupled to a multi-collector ICP-MS (Nu Instruments HR). Ablation in a He atmosphere was either spot-wise or raster-wise according to the zircon CL zonation patterns. Spot analyses were 15-25 $\mu \mathrm{m}$ in diameter, whereas rastering line widths were $10-15 \mu \mathrm{m}$ with a rastering speed of $5 \mu \mathrm{m} / \mathrm{sec}$. The calculated ${ }^{206} \mathrm{~Pb} /{ }^{238} \mathrm{U}$ and ${ }^{207} \mathrm{~Pb} /{ }^{206} \mathrm{~Pb}$ intercept values were corrected for mass discrimination from analyses of standards 91,500 (Wiedenbeck et al. 1995) and Plešovice (Sláma et al. 2006) measured during the analytical session. The correction utilises regression of standard measurements by a quadratic function. A common $\mathrm{Pb}$ correction was applied to the final data using the apparent ${ }^{207} \mathrm{~Pb} /{ }^{206} \mathrm{~Pb}$ age and the Stacey and
Kramers (1975) $\mathrm{Pb}$ evolution model. The final U/Pb ages were calculated with $2 \sigma$ errors using the Isoplot/Ex program, version 3.00 (Ludwig 2003). For comparative studies we also use geochronological data, obtained in the same laboratory and analytical conditions (Gawęda 2008; Burda and Gaweda 2009; Burda et al. 2011, 2013a, b).

\section{Results}

\section{Petrographical characteristics of granitoids}

The tongue-shaped Tatra granitoid intrusion is composed of layered and non-layered series (Fig. 2a, b), intercalating each other in different proportions. Magmatic foliation and lineation are evident in all samples and point out to top-to-SE magma movement, concordant with the metamorphic foliation of host rocks (Kohut and Janak 1994; Gawęda and Szopa 2011). Among non-layered granitoids two granitoid types: porphyritic and equigranular types can be distinguished, medium- to coarse-grained, both showing oriented fabric (Fig. 2b, c). Cumulative textures are also common (Gawęda and Szopa 2011; Szopa et al. 2013). The granitoids of High Tatra type show pronounced layering on different scales (from centimetres to metres in thickness; Gawęda and Szopa 2011).

\section{Sample description}

\section{"Common Tatra" granites}

The Koszysta granitoid (KOS) is a non-layered, equigranular body with an oriented fabric. The mineral components are idiomorphic to subidiomorphic plagioclase $\left(\mathrm{An}_{25-10}\right)$, showing the sharp compositional discontinuities $\left(\mathrm{An}_{38-35}\right)$ called "calcic spikes" (see definition by Hibbard 1991) and interstitial alkali feldspar with $\mathrm{Ba}$ contents in the range 1-3 mol.\%, quartz, biotite with $(\mathrm{Fe} / \mathrm{Fe}+\mathrm{Mg}+\mathrm{Mn})$ ratio (\#fm) in the range 0.53-0.55 and $\mathrm{Ti} 0.36-0.43$ [a.p.f.u], and magmatic muscovite with $\mathrm{Ti}=0.10-0.14$ [a.p.f.u.] (Table 1). Accessories are fluorapatite and zircon.

The granitoid from Wołowiec Mt (WG) is strongly sheared and contains metapelitic xenoliths; it represents the border zone of the intrusion. The primary major components are: quartz, plagioclase $\mathrm{An}_{25-20}, \mathrm{~K}$-feldspar and biotite $(\# \boldsymbol{f m}=0.57-0.62$; Table 1$)$ showing secondary alteration. Accessory components are ilmenite, fluorapatite, zircon, monazite-(Ce) and garnet $\left(\mathrm{Alm}_{66} \mathrm{Spess}_{22} \mathrm{Py}_{9} \mathrm{Grs}_{3}\right)$. Secondary muscovite is abundant.

The Rohač Płaczliwy (RP) porphyritic granitoid shows well-developed magmatic foliation and lineation. K-feldspar porphyrocrysts, showing internal zonation, contain inclusions of older K-feldspars and plagioclases $\mathrm{An}_{25-18}$ 
Table 3 Chemical composition and selected petrological indices of the whole-rock samples classified as common Tatra granites

\begin{tabular}{|c|c|c|c|c|c|c|c|c|}
\hline \multirow[t]{2}{*}{ Sample no. } & \multirow[t]{2}{*}{ LoD } & \multicolumn{2}{|c|}{ Koszysta } & \multirow{2}{*}{$\begin{array}{l}\text { Wołowiec } \\
\text { WG }\end{array}$} & \multicolumn{2}{|l|}{ Rohač } & \multicolumn{2}{|l|}{ Łomnica } \\
\hline & & KOS-1 & KOS-2 & & RP-1 & RP-2 & LOM-1 & LOM-2 \\
\hline \multicolumn{9}{|l|}{$w t \%$} \\
\hline $\mathrm{SiO}_{2}$ & 0.01 & 69.35 & 70.21 & 75.06 & 70.26 & 74.13 & 70.79 & 69.86 \\
\hline $\mathrm{TiO}_{2}$ & 0.01 & 0.33 & 0.37 & 0.36 & 0.29 & 0.40 & 0.65 & 0.36 \\
\hline $\mathrm{Al}_{2} \mathrm{O}_{3}$ & 0.01 & 16.25 & 15.45 & 14.80 & 15.92 & 14.03 & 15.78 & 15.78 \\
\hline $\mathrm{Fe}_{2} \mathrm{O}_{3 \mathrm{~T}}$ & 0.04 & 2.70 & 2.79 & 0.91 & 2.46 & 2.25 & 2.28 & 2.29 \\
\hline $\mathrm{MnO}$ & 0.01 & 0.04 & 0.05 & 0.57 & 0.03 & 0.04 & 0.02 & 0.03 \\
\hline $\mathrm{MgO}$ & 0.01 & 0.89 & 1.10 & 0.38 & 0.86 & 0.43 & 0.71 & 0.74 \\
\hline $\mathrm{CaO}$ & 0.01 & 2.82 & 1.29 & 0.22 & 2.80 & 2.08 & 2.68 & 2.00 \\
\hline $\mathrm{Na}_{2} \mathrm{O}$ & 0.01 & 4.70 & 4.43 & 0.90 & 4.42 & 4.30 & 4.46 & 4.22 \\
\hline $\mathrm{K}_{2} \mathrm{O}$ & 0.01 & 1.88 & 3.02 & 4.61 & 1.79 & 1.47 & 2.06 & 3.73 \\
\hline $\mathrm{P}_{2} \mathrm{O}_{5}$ & 0.01 & 0.12 & 0.15 & 0.14 & 0.13 & 0.05 & 0.11 & 0.11 \\
\hline LOI & - & 0.70 & 1.10 & 2.00 & 0.90 & 1.10 & 0.70 & 1.10 \\
\hline Total & & 99.78 & 99.96 & 99.95 & 99.86 & 100.28 & 100.24 & 100.22 \\
\hline \multicolumn{9}{|l|}{ ppm } \\
\hline $\mathrm{Sr}$ & 0.5 & 687.8 & 414.7 & 29.2 & 659.5 & 458.2 & 635.0 & 552.5 \\
\hline $\mathrm{Ba}$ & 1.0 & 893 & 859 & 299 & 576 & 258 & 833 & 1228 \\
\hline $\mathrm{Rb}$ & 0.1 & 37.5 & 82.0 & 151.4 & 45.1 & 51.0 & 47.6 & 85.7 \\
\hline Th & 0.2 & 6.7 & 11.2 & 9.4 & 7.3 & 7.8 & 6.4 & 13.3 \\
\hline $\mathrm{U}$ & 0.1 & 0.9 & 2.1 & 2.5 & 1.3 & 1.2 & 0.8 & 1.3 \\
\hline $\mathrm{Ga}$ & 0.5 & 17.0 & 20.0 & 17.9 & 19.8 & 16.6 & 19.0 & 19.2 \\
\hline $\mathrm{Ni}$ & 0.1 & 6.1 & 4.8 & 7.5 & 1.8 & 5.0 & 3.1 & 3.3 \\
\hline $\mathrm{Cr}$ & 5.0 & 89.0 & 40.0 & 48.0 & 35.0 & 48.0 & 30.0 & 39.0 \\
\hline $\mathrm{Zr}$ & 0.1 & 131.9 & 122.0 & 95.8 & 153.9 & 125.9 & 138.5 & 107.7 \\
\hline $\mathrm{Hf}$ & 0.1 & 4.3 & 3.6 & 3.1 & 4.6 & 3.9 & 4.6 & 3.5 \\
\hline Y & 0.1 & 6.5 & 15.2 & 20.6 & 8.5 & 5.1 & 11.3 & 8.4 \\
\hline $\mathrm{Nb}$ & 0.1 & 2.8 & 7.9 & 5.8 & 4.6 & 5.8 & 4.8 & 6.0 \\
\hline $\mathrm{Ta}$ & 0.1 & 0.1 & 0.4 & 0.4 & 0.4 & 0.7 & 0.3 & 0.5 \\
\hline $\mathrm{La}$ & 0.1 & 21.8 & 27.9 & 17.1 & 30.7 & 28.0 & 29.2 & 29.8 \\
\hline $\mathrm{Ce}$ & 0.1 & 46.3 & 58.8 & 35.9 & 62.5 & 56.7 & 55.3 & 62.9 \\
\hline $\operatorname{Pr}$ & 0.02 & 5.20 & 6.78 & 3.97 & 6.99 & 6.62 & 6.30 & 7.16 \\
\hline $\mathrm{Nd}$ & 0.30 & 21.7 & 24.6 & 14.5 & 26.1 & 23.5 & 24.3 & 28.1 \\
\hline Sm & 0.05 & 3.42 & 4.49 & 3.60 & 4.70 & 4.30 & 4.10 & 4.73 \\
\hline Eu & 0.02 & 0.92 & 0.76 & 0.82 & 1.07 & 0.92 & 0.99 & 1.07 \\
\hline $\mathrm{Gd}$ & 0.05 & 2.65 & 3.27 & 3.13 & 2.57 & 2.81 & 2.32 & 3.15 \\
\hline $\mathrm{Tb}$ & 0.01 & 0.30 & 0.53 & 0.79 & 0.44 & 0.32 & 0.43 & 0.43 \\
\hline Dy & 0.05 & 1.54 & 2.87 & 3.76 & 1.77 & 1.31 & 2.19 & 1.95 \\
\hline Ho & 0.02 & 0.26 & 0.53 & 0.62 & 0.26 & 0.18 & 0.40 & 0.28 \\
\hline $\mathrm{Er}$ & 0.03 & 0.67 & 1.41 & 1.69 & 0.69 & 0.41 & 1.19 & 0.67 \\
\hline $\mathrm{Tm}$ & 0.01 & 0.10 & 0.21 & 0.23 & 0.09 & 0.07 & 0.15 & 0.09 \\
\hline $\mathrm{Yb}$ & 0.05 & 0.64 & 1.30 & 1.27 & 0.69 & 0.42 & 0.84 & 0.56 \\
\hline $\mathrm{Lu}$ & 0.01 & 0.10 & 0.19 & 0.19 & 0.10 & 0.06 & 0.12 & 0.08 \\
\hline ASI & & 1.11 & 1.23 & 2.15 & 1.14 & 1.14 & 1.11 & 1.10 \\
\hline $\mathrm{Rb} / \mathrm{Sr}$ & & 0.05 & 0.20 & 5.18 & 0.07 & 0.11 & 0.07 & 0.16 \\
\hline $\mathrm{Nd} / \mathrm{Th}$ & & 3.24 & 2.20 & 1.54 & 3.57 & 22.10 & 3.80 & 2.11 \\
\hline$\Sigma$ REE & & 105.6 & 133.64 & 87.57 & 138.67 & 125.62 & 127.83 & 140.97 \\
\hline $\mathrm{Eu} / \mathrm{Eu}^{*}$ & & 0.93 & 0.61 & 0.75 & 0.94 & 0.81 & 0.98 & 0.85 \\
\hline $\mathrm{Ce}_{\mathrm{N}} / \mathrm{Yb}_{\mathrm{N}}$ & & 19.93 & 12.46 & 7.79 & 24.96 & 37.20 & 18.14 & 30.95 \\
\hline$T_{\mathrm{Zr}}\left({ }^{\circ} \mathrm{C}\right)$ & & 794 & 804 & 817 & 779 & 800 & 801 & 782 \\
\hline
\end{tabular}

$L o D$ limits of detection, $L O I$ lost of ignition

$\mathrm{Eu} / \mathrm{Eu}^{*}=\mathrm{Eu} / \sqrt{ } \mathrm{Sm} \cdot \mathrm{Gd}$

ASI $=\mathrm{Al}_{2} \mathrm{O}_{3} /\left(\mathrm{CaO}+\mathrm{Na}_{2} \mathrm{O}+\mathrm{K}_{2} \mathrm{O}-3.33 \mathrm{P}_{2} \mathrm{O}_{5}\right)$ (in molecular values)

$T_{\mathrm{Zr}}=$ temperature calculated according to Watson and Harrison (1983) procedure 
Table 4 Chemical composition and selected petrological indices of the whole-rock samples classified as High Tatra granites

\begin{tabular}{|c|c|c|c|c|c|c|c|}
\hline \multirow[t]{2}{*}{ Sample no. } & \multicolumn{7}{|l|}{ WPP } \\
\hline & LoD & WP-G & WP-Z & WPP-1 & WPP-2 & WPP-3 & WPP-4 \\
\hline \multicolumn{8}{|l|}{$w t \%$} \\
\hline $\mathrm{SiO}_{2}$ & 0.01 & 71.33 & 70.55 & 73.89 & 72.39 & 61.07 & 62.9 \\
\hline $\mathrm{TiO}_{2}$ & 0.01 & 0.27 & 0.29 & 0.27 & 1.23 & 0.77 & 0.65 \\
\hline $\mathrm{Al}_{2} \mathrm{O}_{3}$ & 0.01 & 15.59 & 15.68 & 13.19 & 15.16 & 17.91 & 18.87 \\
\hline $\mathrm{Fe}_{2} \mathrm{O}_{3 \mathrm{~T}}$ & 0.04 & 1.87 & 2.37 & 2.24 & 1.60 & 5.89 & 3.23 \\
\hline $\mathrm{MnO}$ & 0.01 & 0.05 & 0.06 & 0.03 & 0.11 & 0.09 & 0.02 \\
\hline $\mathrm{MgO}$ & 0.01 & 0.79 & 0.87 & 0.56 & 0.79 & 2.73 & 1.92 \\
\hline $\mathrm{CaO}$ & 0.01 & 1.16 & 1.12 & 1.29 & 0.97 & 3.33 & 1.67 \\
\hline $\mathrm{Na}_{2} \mathrm{O}$ & 0.01 & 4.76 & 4.81 & 3.29 & 4.39 & 4.31 & 4.71 \\
\hline $\mathrm{K}_{2} \mathrm{O}$ & 0.01 & 2.63 & 2.70 & 3.78 & 3.11 & 2.33 & 3.56 \\
\hline $\mathrm{P}_{2} \mathrm{O}_{5}$ & 0.01 & 0.11 & 0.04 & 0.06 & 0.10 & 0.36 & 0.25 \\
\hline LOI & - & 1.20 & 1.40 & 1.30 & 1.30 & 1.30 & 2.3 \\
\hline Total & & 99.76 & 99.89 & 99.90 & 101.15 & 100.09 & 100.08 \\
\hline \multicolumn{8}{|l|}{ ppm } \\
\hline $\mathrm{Sr}$ & 0.5 & 456.8 & 400.2 & 251.2 & 307.2 & 735.2 & 319.5 \\
\hline $\mathrm{Ba}$ & 1.0 & 1155 & 492 & 752 & 687 & 682 & 1094 \\
\hline $\mathrm{Rb}$ & 0.1 & 64.6 & 73.0 & 67.4 & 75.3 & 107.5 & 91.9 \\
\hline Th & 0.2 & 6.6 & 10.1 & 5.0 & 5.3 & 19.3 & 15.9 \\
\hline $\mathrm{U}$ & 0.1 & 1.0 & 2.2 & 1.0 & 0.6 & 4.0 & 1.7 \\
\hline $\mathrm{Ga}$ & 0.5 & 18.5 & 20.3 & 15.5 & 17.8 & 24.4 & 22.7 \\
\hline $\mathrm{Ni}$ & 0.1 & 1.9 & 6.7 & 3.2 & 6.6 & 6.0 & 5.0 \\
\hline $\mathrm{Cr}$ & 5.0 & 57.0 & 86.0 & 51.0 & 72.0 & 47.9 & 20.6 \\
\hline $\mathrm{Zr}$ & 0.1 & 138.9 & 107.5 & 66.5 & 89.8 & 222.0 & 205.8 \\
\hline $\mathrm{Hf}$ & 0.1 & 4.0 & 3.6 & 2.1 & 2.9 & 3.6 & 6.0 \\
\hline Y & 0.1 & 11.5 & 10.0 & 11.6 & 10.2 & 22.0 & 16.8 \\
\hline $\mathrm{Nb}$ & 0.1 & 4.5 & 8.6 & 4.8 & 5.0 & 8.6 & 8.5 \\
\hline $\mathrm{Ta}$ & 0.1 & 0.3 & 1.0 & 0.3 & 0.5 & 0.6 & 0.5 \\
\hline $\mathrm{La}$ & 0.1 & 23.9 & 15.6 & 14.1 & 17.8 & 65.6 & 49.7 \\
\hline $\mathrm{Ce}$ & 0.1 & 50.9 & 31.2 & 30.8 & 34.5 & 129.6 & 104.6 \\
\hline $\operatorname{Pr}$ & 0.02 & 5.76 & 3.65 & 3.83 & 4.26 & 14.13 & 11.98 \\
\hline $\mathrm{Nd}$ & 0.30 & 22.9 & 14.6 & 14.7 & 16.5 & 50.6 & 42.8 \\
\hline $\mathrm{Sm}$ & 0.05 & 3.44 & 2.78 & 2.87 & 3.10 & 8.6 & 8.5 \\
\hline $\mathrm{Eu}$ & 0.02 & 0.92 & 0.61 & 0.69 & 0.64 & 1.58 & 1.69 \\
\hline $\mathrm{Gd}$ & 0.05 & 2.47 & 2.12 & 2.61 & 1.90 & 6.01 & 5.89 \\
\hline $\mathrm{Tb}$ & 0.01 & 0.37 & 0.33 & 0.35 & 0.42 & 0.84 & 0.91 \\
\hline Dy & 0.05 & 1.92 & 1.76 & 2.34 & 1.73 & 4.24 & 3.99 \\
\hline Ho & 0.02 & 0.39 & 0.34 & 0.46 & 0.33 & 0.72 & 0.56 \\
\hline $\mathrm{Er}$ & 0.03 & 1.05 & 0.95 & 1.35 & 0.96 & 1.91 & 1.36 \\
\hline $\mathrm{Tm}$ & 0.01 & 0.16 & 0.15 & 0.20 & 0.13 & 0.30 & 0.2 \\
\hline $\mathrm{Yb}$ & 0.05 & 1.02 & 0.98 & 1.21 & 0.97 & 1.62 & 1.15 \\
\hline $\mathrm{Lu}$ & 0.01 & 0.16 & 0.14 & 0.18 & 0.13 & 0.26 & 0.19 \\
\hline ASI & & 1.25 & 1.27 & 1.13 & 1.25 & 1.21 & 1.34 \\
\hline $\mathrm{Rb} / \mathrm{Sr}$ & & 0.141 & 0.182 & 0.27 & 0.245 & 0.146 & 0.288 \\
\hline $\mathrm{Nd} / \mathrm{Th}$ & & 3.47 & 1.45 & 2.94 & 3.11 & 2.62 & 2.69 \\
\hline$\Sigma$ REE & & 115.36 & 75.21 & 75.69 & 83.37 & 286.01 & 233.52 \\
\hline $\mathrm{Eu} / \mathrm{Eu}^{*}$ & & 0.97 & 0.77 & 0.77 & 0.81 & 0.67 & 0.73 \\
\hline $\mathrm{Ce}_{\mathrm{N}} / \mathrm{Yb}_{\mathrm{N}}$ & & 13.75 & 8.77 & 7.01 & 9.80 & 22.04 & 25.06 \\
\hline$T_{\mathrm{Zr}}\left({ }^{\circ} \mathrm{C}\right)$ & & 755 & 795 & 749 & 782 & 831 & 849 \\
\hline
\end{tabular}

$L o D$ limits of detection, $L O I$ lost of ignition

$\mathrm{Eu} / \mathrm{Eu}^{*}=\mathrm{Eu} / \sqrt{ } \mathrm{Sm} \cdot \mathrm{Gd}$

$\mathrm{ASI}=\mathrm{Al}_{2} \mathrm{O}_{3} /\left(\mathrm{CaO}+\mathrm{Na}_{2} \mathrm{O}+\mathrm{K}_{2} \mathrm{O}-3.33 \mathrm{P}_{2} \mathrm{O}_{5}\right)$ (in molecular values)

$T_{\mathrm{Zr}}=$ temperature calculated according to Watson and Harrison (1983) procedure 


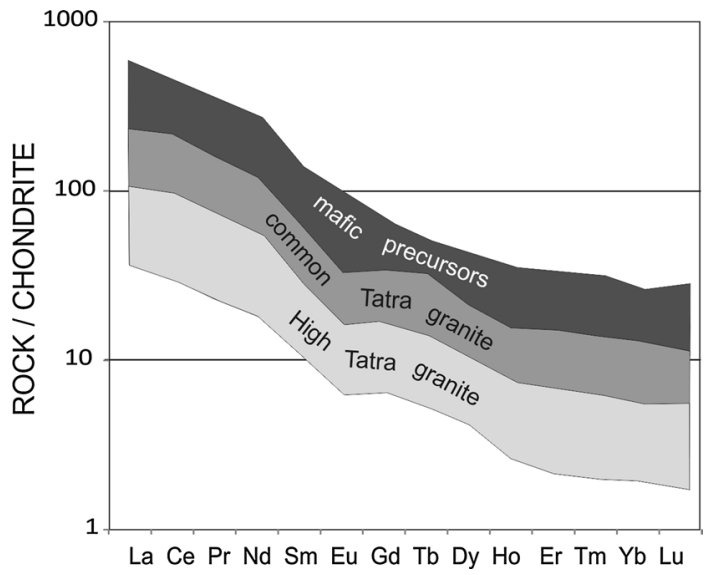

Fig. 5 Chondrite (C1)-normalised REE concentrations of the Tatra granitoids in relation to their mafic precursors (after Gawęda et al. 2005)

(Fig. 3a, b). Biotite with \#fm in the range 0.48-0.52 and $\mathrm{Ti}=0.31-0.39$ [a.p.f.u.] is the typical mafic component, associated with rare $\mathrm{Mg}$ hornblende-tschermakitic hornblende (Table 2). Accessory minerals are composite grains of magnetite-ilmenite intergrowths, fluorapatite, zircon, monazite, allanite. Syn-magmatic and late magmatic deformation is expressed both at outcrop-scale, as magmatic faulting and brecciation (Gawęda and Szopa 2011), and at the microscopic scale, as mineral brecciation and sealing (Fig. 3c) and folding of plagioclase twinning (Fig. 3d).

The homogeneous, non-layered granodiorite-tonalite from Łomnica (LOM) is composed of zoned plagioclase $\left(\mathrm{An}_{28-21}\right)$ with calcic spikes $\left(\mathrm{An}_{38-40}\right)$, K-feldspar zoned with respect to $\mathrm{Ba}$, quartz, magmatic muscovite $\left(\mathrm{TiO}_{2}>1 \mathrm{wt} \%\right)$ and biotite $(\# \boldsymbol{f m}=0.53-0.57$ and $\mathrm{Ti}=0.21-0.36)$. Ilmenite-magnetite intergrowths are commonly observed. Accessories are fluorapatite and zircon. Secondary hematite is often found. The late magmatic deformation resulted in a penetrative foliation.

\section{"High Tatra” granites}

The layered Mięguszowieckie Wierchy-Wielki Piarg (WPP) granitoid shows a concentration of mafic minerals (biotite, ilmenite-magnetite intergrowths, magnetite, ulvospinel) and Ba-rich K-feldspars (up to 7 mol.\% of $\mathrm{Ba}$ [a.p.f.u.]) at the base of 30- to 70-cm-thick layers. Biotite has $\# \mathbf{f m}=0.57-0.63$ and $\mathrm{Ti}=0.30-0.42$ [a.p.f.u.]. Felsic components (feldspar and quartz) are concentrated at the tops of layers. Plagioclase is mostly oligoclase $\left(\mathrm{An}_{28-16}\right)$, and interstitial alkali feldspar is strongly perthitic. A wide range of accessory minerals concentrate usually at the base of the layer: monazite-(Ce), xenotime, fluorapatite, zircon. Secondary titanite and REE-enriched epidote suggest late magmatic oxidation (Gawęda 2009, 2008; Burda et al. 2013b). Antecrysts often form partially resorbed crystals. They are represented by the inclusions of K-feldspars and plagioclases in K-feldspars phenocrysts, partly resorbed "ghost" cores of K-feldspars in matrix K-feldspars, showing different $\mathrm{Ba}$ zonation (Fig. 3e, f) and also xenocrystic quartz crystals (millimetre to centimetre in size; Fig. 3g), present elsewhere in the granitoids. These could be remnants of older granite enclaves (Fig. 2d), disaggregated and resorbed to different extents (Gawęda 2008, 2009; Gawęda and Szopa 2011).

\section{Whole-rock chemistry}

Granitoid rocks of the Tatra Mountains occupy the monzonite-quartz-monzonite_granodiorite-granite fields on a $\mathrm{Na}_{2} \mathrm{O}+\mathrm{K}_{2} \mathrm{O}$ versus $\mathrm{SiO}_{2}$ diagram (Fig. 4a; Middlemost 1985). All samples are peraluminous, with ASI indices ranging from 1.11 to 2.15 and plot as calc-alkaline to high-K calc-alkaline magmas (Tables 3, 4; Fig. 4b). Samples which belong to the so-called common Tatra type granitoids tend to group in the calc-alkaline field, whilst so-called High Tatra type granitoids plot mostly as high$\mathrm{K}$ calc-alkaline rocks (Fig. $4 \mathrm{~b}$ ). $\mathrm{Na}_{2} \mathrm{O}$ prevails over $\mathrm{K}_{2} \mathrm{O}$ (mean $\mathrm{Na}_{2} \mathrm{O} / \mathrm{K}_{2} \mathrm{O}$ ratio of 1.55 ). The $\mathrm{Rb} / \mathrm{Sr}$ ratio is in the range of $0.05-0.27$ (mean value $=0.15$ ), whilst the $\mathrm{Nd} /$ Th ratio ranges from 2.8 to 10.3 (Tables 3,4 ). Chondritenormalised (Sun and McDonough, 1989) REE patterns show relatively flat REE patterns $\left(\mathrm{Ce}_{\mathrm{N}} / \mathrm{Yb}_{\mathrm{N}}=6.32-19.10\right)$ with moderately to weakly negative Eu anomalies (Eu/ $\mathrm{Eu}^{*}=0.56-0.89$; Tables 3, 4; Fig. 5), typical of magmas extracted from a deep crustal source, containing residual garnet (Miller 1985).

Zirconium shows a positive correlation with $\mathrm{SiO}_{2}$ in the mafic granitoid varieties $\left(\mathrm{SiO}_{2}<67 \mathrm{wt} \%\right)$ and a negative correlation in the felsic varieties (Fig. 4c). Whole-rock zirconium contents in relatively homogeneous rock samples were used to calculate the magma temperature according to Watson and Harrison (1983) procedure, using equation $T_{\mathrm{Zr}}=12,900 /\left[2.95+0.85 M+\ln \left(496,000 / \mathrm{Zr}_{\text {melt }}\right)\right]$, where $M$ is a compositional factor. The analysed samples yielded temperatures in the range of $873-749{ }^{\circ} \mathrm{C}$ (Tables 3,4 ).

\section{Geochronology}

Zircon from five granitoid samples of the Tatra massif was analysed using LA-MC-ICP-MS. The results are summarised in Table 5.

(a) Zircon crystals from sample KOS are colourless to light yellow and vary in length from ca. 100 to $250 \mu \mathrm{m}$. All zircons are perfectly euhedral, differing only in their aspect ratios which range from 1:2 to $1: 4$. 
Table 5 LA-MC-ICP-MS U-Pb zircon data from Tatra Mountains granitoids (samples given from the oldest computed age: KOS, WG, RP, LOM, WPP)

\begin{tabular}{|c|c|c|c|c|c|c|c|c|c|c|c|c|}
\hline \multirow[t]{2}{*}{ File name } & \multicolumn{4}{|c|}{$\begin{array}{l}\text { Final blank corrected intensities } \\
\text { (in V) }\end{array}$} & \multicolumn{7}{|c|}{ Final mass bias and common $\mathrm{Pb}$ corrected ratios } & \multirow[t]{2}{*}{$\begin{array}{l}\text { Concordia } \\
\text { age (Ma) }\end{array}$} \\
\hline & ${ }^{204} \mathrm{~Pb}^{*}$ & ${ }^{206} \mathrm{~Pb}^{\#}$ & ${ }^{207} \mathrm{~Pb}^{\#}$ & ${ }^{238} U^{\#}$ & ${ }^{207} \mathrm{~Pb}^{/ 206} \mathrm{~Pb}$ & 2RSE $(\%)$ & ${ }^{207} \mathrm{~Pb}^{/ 235} \mathrm{U}$ & 2RSE $(\%)$ & ${ }^{206} \mathrm{~Pb}^{1238} \mathrm{U}$ & 2RSE (\%) & $\overline{\text { Rho }}$ & \\
\hline \multicolumn{13}{|l|}{ Sample KOS } \\
\hline KOS_III_02 & 0.796 & 0.501 & 0.019 & 15.09 & 0.0560 & 22.82 & 0.4610 & 52.66 & 0.0596 & 52.87 & 0.50 & \\
\hline KOS_III_05 & 1.234 & 0.823 & 0.030 & 25.69 & 0.0516 & 30.72 & 0.4125 & 33.30 & 0.0580 & 6.35 & 0.10 & \\
\hline KOS_III_08 & 0.863 & 0.426 & 0.017 & 13.05 & 0.0591 & 17.94 & 0.4988 & 21.03 & 0.0621 & 16.88 & 0.40 & \\
\hline KOS_III_11 & 0.734 & 0.214 & 0.008 & 6.38 & 0.0542 & 17.69 & 0.4226 & 65.47 & 0.0571 & 47.85 & 0.37 & \\
\hline KOS_III_12 & 0.791 & 0.143 & 0.005 & 4.68 & 0.0529 & 20.63 & 0.4176 & 22.48 & 0.0564 & 22.42 & 0.50 & $368.3 \pm 9.4$ \\
\hline KOS_III_14 & 1.187 & 0.561 & 0.020 & 17.80 & 0.0536 & 3.79 & 0.4324 & 6.30 & 0.0585 & 4.20 & 0.33 & \\
\hline KOS_III_15 & 0.608 & 0.396 & 0.014 & 12.23 & 0.0545 & 8.73 & 0.4414 & 15.08 & 0.0586 & 22.67 & 0.75 & \\
\hline KOS_III_16 & 0.880 & 0.496 & 0.018 & 16.06 & 0.0528 & 20.53 & 0.4226 & 26.96 & 0.0574 & 15.92 & 0.30 & \\
\hline KOS_III_17 & 0.714 & 0.314 & 0.013 & 10.99 & 0.0614 & 12.78 & 0.4619 & 10.68 & 0.0556 & 11.45 & 0.54 & \\
\hline KOS_III_19 & 1.332 & 0.867 & 0.031 & 27.87 & 0.0533 & 19.27 & 0.4326 & 29.03 & 0.0597 & 10.94 & 0.19 & \\
\hline KOS_III_20 & 0.921 & 1.352 & 0.046 & 40.99 & 0.0540 & 8.81 & 0.4595 & 15.46 & 0.0624 & 11.78 & 0.38 & \\
\hline \multicolumn{13}{|l|}{ Sample $W G$} \\
\hline WG2_IIIa_01/1 & 1.513 & 2.256 & 0.186 & 124.37 & 0.0549 & 1.8 & 0.4297 & 3.5 & 0.0578 & 3.1 & 0.52 & \\
\hline WG2_IIIa_03 & 0.443 & 0.674 & 0.053 & 28.84 & 0.0539 & 8.8 & 0.4315 & 17.6 & 0.0586 & 15.6 & 0.57 & \\
\hline WG2_IIIa_04 & 0.742 & 0.819 & 0.067 & 37.75 & 0.0533 & 3.1 & 0.4357 & 6.1 & 0.0592 & 5.4 & 0.44 & \\
\hline WG2_IIIa_05 & 0.722 & 0.811 & 0.066 & 37.15 & 0.0531 & 3.1 & 0.4314 & 5.1 & 0.0582 & 5.3 & 0.42 & $364.7 \pm 5.3$ \\
\hline WG2_IIIa_06 & 0.721 & 0.513 & 0.043 & 24.00 & 0.0552 & 3.4 & 0.4428 & 6.6 & 0.0583 & 5.8 & 0.52 & \\
\hline WG2_IIIa_07 & 0.526 & 0.619 & 0.052 & 27.87 & 0.0551 & 3.8 & 0.4555 & 7.4 & 0.0598 & 6.6 & 0.48 & \\
\hline WG2_IIIa_09 & 0.533 & 0.791 & 0.065 & 35.88 & 0.0538 & 2.0 & 0.4277 & 3.9 & 0.0576 & 3.4 & 0.45 & \\
\hline WG2_IIIa_01/2 & 0.734 & 0.249 & 0.019 & 7.40 & 0.0532 & 3.2 & 0.6543 & 6.2 & 0.0851 & 5.5 & 0.28 & $518 \pm 21$ \\
\hline \multicolumn{13}{|l|}{ Sample RP } \\
\hline RP_IId_13/1 & 0.755 & 0.704 & 0.039 & 23.77 & 0.0527 & 36.68 & 0.4016 & 8.04 & 0.0563 & 36.62 & 0.27 & \\
\hline RP_IId_13/2 & 0.981 & 0.526 & 0.041 & 22.88 & 0.0534 & 32.77 & 0.4129 & 7.13 & 0.0557 & 32.79 & 0.52 & \\
\hline RP_IId_14/1 & 0.895 & 0.562 & 0.046 & 25.34 & 0.0530 & 5.35 & 0.4187 & 10.37 & 0.0561 & 9.17 & 0.49 & \\
\hline RP_IId_14/2 & 1.206 & 0.208 & 0.017 & 9.21 & 0.0574 & 30.30 & 0.4231 & 6.60 & 0.0541 & 30.31 & 0.38 & \\
\hline RP_IId_15/1 & 1.138 & 0.208 & 0.019 & 10.53 & 0.0599 & 10.17 & 0.4261 & 20.16 & 0.0565 & 17.73 & 0.45 & $360.2 \pm 5.5$ \\
\hline RP_IId_15/2 & 0.701 & 0.386 & 0.032 & 17.68 & 0.0568 & 4.05 & 0.4266 & 7.85 & 0.0585 & 6.94 & 0.65 & \\
\hline RP_IId_16 & 0.742 & 0.819 & 0.067 & 37.75 & 0.0533 & 3.1 & 0.4357 & 6.1 & 0.0592 & 5.4 & 0.44 & \\
\hline RP_IId_17 & 0.533 & 0.791 & 0.065 & 35.88 & 0.0538 & 2.0 & 0.4277 & 3.9 & 0.0576 & 3.4 & 0.45 & \\
\hline RP_IId_19/1 & 0.902 & 0.500 & 0.041 & 22.26 & 0.0558 & 29.81 & 0.4285 & 6.49 & 0.0556 & 29.80 & 0.30 & \\
\hline RP_IId_19/2 & 2.015 & 0.261 & 0.024 & 11.51 & 0.0577 & 2.66 & 0.4285 & 5.17 & 0.0580 & 4.56 & 0.41 & \\
\hline RP_IId_21 & 1.369 & 1.236 & 0.072 & 36.64 & 0.0564 & 14.44 & 0.4325 & 27.40 & 0.0569 & 21.92 & 0.50 & \\
\hline \multicolumn{13}{|l|}{ Sample LOM } \\
\hline LOM_II_3/1 & 1.243 & 2.182 & 0.116 & 67.37 & 0.0546 & 9.6 & 0.4440 & 21.2 & 0.0598 & 19.8 & 0.49 & \\
\hline LOM_II_10 & 1.013 & 2.085 & 0.127 & 68.49 & 0.0556 & 9.6 & 0.4340 & 19.2 & 0.0578 & 17.8 & 0.47 & \\
\hline LOM_III_05 & 1.172 & 1.916 & 0.113 & 68.53 & 0.0538 & 3.4 & 0.4074 & 6.7 & 0.0563 & 5.9 & 0.41 & \\
\hline LOM_III_08/1 & 1.361 & 1.816 & 0.118 & 65.72 & 0.0548 & 3.3 & 0.4174 & 6.7 & 0.0566 & 5.6 & 0.39 & \\
\hline LOM_III_08/2 & 1.271 & 1.741 & 0.106 & 62.37 & 0.0544 & 4.6 & 0.4103 & 9.0 & 0.0552 & 7.9 & 0.32 & $355.2 \pm 8.2$ \\
\hline LOM_III_09/1 & 1.341 & 1.623 & 0.115 & 61.33 & 0.0554 & 4.6 & 0.4203 & 8.5 & 0.0562 & 8.7 & 0.38 & \\
\hline LOM_III_09/2 & 1.461 & 1.968 & 0.127 & 67.81 & 0.0561 & 13.8 & 0.4520 & 26.6 & 0.0574 & 23.6 & 0.40 & \\
\hline LOM_III_11 & 1.341 & 1.878 & 0.132 & 66.70 & 0.0571 & 13.8 & 0.4420 & 21.6 & 0.0563 & 21.6 & 0.38 & \\
\hline LOM_III_14/1 & 1.376 & 0.687 & 0.044 & 24.49 & 0.0580 & 5.9 & 0.4529 & 11.5 & 0.0575 & 10.1 & 0.40 & \\
\hline LOM_III_14/2 & 1.453 & 0.674 & 0.042 & 26.25 & 0.0575 & 4.9 & 0.4429 & 10.5 & 0.0565 & 9.1 & 0.38 & \\
\hline LOM_II_03/2 & 1.544 & 2.101 & 0.135 & 65.75 & 0.0577 & 6.9 & 0.5064 & 13.6 & 0.0637 & 11.9 & 0.41 & \\
\hline LOM_II_10/2 & 1.211 & 0.948 & 0.057 & 29.07 & 0.0649 & 11.1 & 0.5736 & 21.9 & 0.0618 & 18.8 & 0.38 & $426 \pm 25$ \\
\hline
\end{tabular}


Table 5 continued

\begin{tabular}{|c|c|c|c|c|c|c|c|c|c|c|c|c|}
\hline \multirow[t]{2}{*}{ File name } & \multicolumn{4}{|c|}{$\begin{array}{l}\text { Final blank corrected intensities } \\
\text { (in V) }\end{array}$} & \multicolumn{7}{|c|}{ Final mass bias and common $\mathrm{Pb}$ corrected ratios } & \multirow[t]{2}{*}{$\begin{array}{l}\text { Concordia } \\
\text { age }(\mathrm{Ma})\end{array}$} \\
\hline & ${ }^{204} \mathrm{~Pb}^{*}$ & ${ }^{206} \mathrm{~Pb}^{\#}$ & ${ }^{207} \mathrm{~Pb}^{\#}$ & ${ }^{238} \mathrm{U}^{\#}$ & ${ }^{207} \mathrm{~Pb}^{1206} \mathrm{~Pb}$ & 2RSE (\%) & ${ }^{207} \mathrm{~Pb}^{/ 235} \mathrm{U}$ & 2RSE (\%) & ${ }^{206} \mathrm{~Pb}^{1238} \mathrm{U}$ & $2 \operatorname{RSE}(\%)$ & $\overline{\text { Rho }}$ & \\
\hline$\overline{\text { LOM_II_10/3 }}$ & 1.525 & 0.450 & 0.032 & 13.32 & 0.0628 & 6.4 & 0.5944 & 12.4 & 0.0691 & 10.9 & 0.32 & \\
\hline \multicolumn{13}{|l|}{ Sample WPP } \\
\hline WPP_IIc_01 & 1.221 & 0.609 & 0.026 & 16.11 & 0.0544 & 3.0 & 0.4249 & 5.9 & 0.0551 & 5.6 & 0.39 & \\
\hline WPP_IIc_05 & 0.997 & 0.290 & 0.012 & 7.52 & 0.0549 & 3.3 & 0.4080 & 6.5 & 0.0543 & 5.6 & 0.19 & \\
\hline WPP_IIc_06/1 & 0.567 & 0.173 & 0.007 & 4.20 & 0.0529 & 3.4 & 0.4024 & 6.6 & 0.0545 & 5.8 & 0.23 & \\
\hline WPP_IIc_12/1 & 0.592 & 0.425 & 0.018 & 10.69 & 0.0526 & 3.0 & 0.4018 & 5.8 & 0.0557 & 5.2 & 0.28 & \\
\hline WPP_IIc_12/2 & 1.513 & 2.256 & 0.186 & 124.37 & 0.0549 & 1.8 & 0.4007 & 3.5 & 0.0538 & 3.1 & 0.52 & $345.6 \pm 3.7$ \\
\hline WPP_IIc_13 & 0.984 & 0.413 & 0.017 & 9.97 & 0.0540 & 1.6 & 0.4101 & 3.0 & 0.0550 & 2.7 & 0.44 & \\
\hline WPP_IIc_14 & 1.271 & 1.741 & 0.106 & 62.37 & 0.0544 & 4.6 & 0.4103 & 9.0 & 0.0552 & 7.9 & 0.32 & \\
\hline WPP_IIc_17 & 1.110 & 0.413 & 0.017 & 10.40 & 0.0545 & 2.8 & 0.4112 & 5.4 & 0.0545 & 4.8 & 0.17 & \\
\hline WPP_IIc_19 & 0.836 & 0.708 & 0.029 & 17.36 & 0.0531 & 2.7 & 0.4087 & 5.4 & 0.0545 & 5.1 & 0.48 & \\
\hline WPP_IIc_21/1 & 1.172 & 1.916 & 0.113 & 68.53 & 0.0538 & 3.4 & 0.4074 & 6.7 & 0.0563 & 5.9 & 0.41 & \\
\hline WPP_IIc_21/2 & 1.158 & 0.375 & 0.017 & 9.88 & 0.0552 & 2.3 & 0.4072 & 4.5 & 0.0554 & 4.3 & 0.34 & \\
\hline WPP_IIc_06/2 & 0.495 & 1.097 & 0.049 & 20.57 & 0.0555 & 2.8 & 0.5738 & 5.4 & 0.0747 & 4.8 & 0.45 & $462 \pm 18$ \\
\hline WPP_IIc_21/3 & 0.565 & 0.237 & 0.011 & 3.94 & 0.0576 & 2.8 & 0.6809 & 5.4 & 0.0875 & 4.8 & 0.28 & $533 \pm 19$ \\
\hline
\end{tabular}

2RSE 2-sigma relative standard error (in \%), Rho the error correlation between the 206/238 and 207/235 ratios

* Final blank corrected intensities in $\mu \mathrm{V},{ }^{\#}$ final blank corrected intensities in $\mathrm{mV}$

CL investigations show that the short and normal prismatic crystals usually exhibit two different domains: a homogeneous to weakly growth-zoned internal part (core) surrounded by a younger rim with fine to broad oscillatory zoning, displaying variable CL intensities. Cores are sub-rounded and the contacts with surrounding growth-zoned rims are irregular. The long prismatic crystals (single-phase crystals) show only oscillatory zoning (Fig. 6). One LA-MC-ICP-MS U-Pb measurement for each of 11 zircon crystals was made (Table $5 \mathrm{KOS}$ ). All analyses from the oscillatory-zoned zircon zones yield a concordia age of $368 \pm 9 \mathrm{Ma}$ (MSWD $=0.7$, Fig. 7a), which we interpret as the best estimate of the age of crystallisation.

(b) Zircon grains from sample WG are euhedral, up to $300 \mu \mathrm{m}$ long, and are clear to yellow and greenish yellow in colour. CL investigations show that oscillatory zoning is the prominent textural feature, with growth bands varying between fine and broad within individual grains (Fig. 8). Luminescence of growth zoning is variable and mostly moderate. Sporadically, zircons are composed of cores with well-developed oscillatory zoning, indicative of original growth from melt. Eight LA-MC-ICP-MS U-Pb measurements on seven crystals were made (Table $5 \mathrm{WG}$ ). All data points are concordant within the assigned error. Seven analyses from the oscillatory-zoned zircon zones yield a concordia age of $364 \pm 5 \mathrm{Ma}(\mathrm{MSWD}=0.1$, Fig. $7 \mathrm{~b})$. One inherited core plot as $518 \pm 21 \mathrm{Ma}$ (Fig. 7b; Table 5 WG).

(c) Zircon crystals from sample RP are typically 200$300 \mu \mathrm{m}$ in length, euhedral, normal to long prismatic, with aspect ratios of 1:2-1:4. The zircons vary from very clear and colourless to greenish, yellow, reddishbrown to nearly black. Most grains exhibit the oscillatory zoning characteristic of magmatic growth (singlephase crystals). Some grains have brighter CL interior domains when compared with the external parts. These interior domains have boundaries parallel to external oscillatory zoning (Fig. 9; grains RP_IId_15, RP_ IId_19), and they are not considered to be inherited cores. Some crystals have an inherited component surrounded by an oscillatory-zoned rim (Fig. 9). Eleven LA-MC-ICP-MS U-Pb measurements were made in seven zircon grains (Table $5 \mathrm{RP}$ ) and yield a concordia age of $360 \pm 5 \mathrm{Ma}(\mathrm{MSWD}=0.39$; Fig. 7c). Since all these zircons have faint oscillatory zoning and pyramidal terminations, this age is considered to be the crystallisation age.

(d) The zircon grains from sample LOM are euhedral, normal to long prismatic crystals with aspect ratios from 1:2 to 1:4. Grain size varies in length from ca. 50 to $250 \mu \mathrm{m}$. Zircons appear clear, colourless to pink. Most grains are characterised by well-developed oscillatory zoning, ranging from fine to broad, and display variable luminescence. The majority of grains lack discern- 


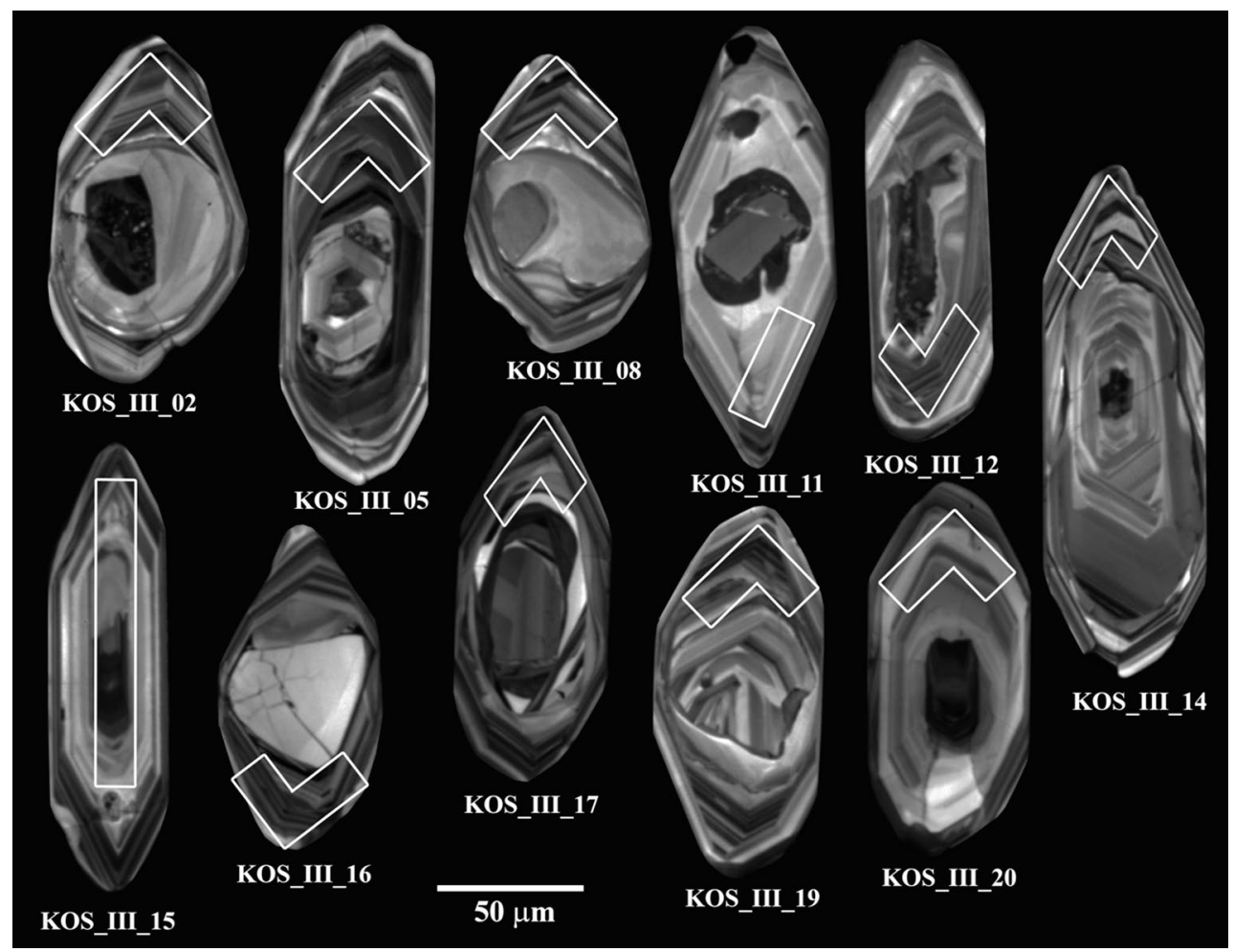

Fig. 6 Cathodoluminescence (CL) images of zircon crystals from a KOS sample (Koszysta granitoid, High Tatra Mountains)

ible, inherited cores, but where preserved, cores are zoned and distinct from the enclosing rims by virtue of luminescence and truncated zoning. The cores show well-developed oscillatory zoning indicating an igneous source. Thirteen LA-MC-ICP-MS U-Pb measurements on seven crystals were made (Fig. 10). Ten analyses from the oscillatory-zoned zircon yield a concordia age of $355 \pm 8 \mathrm{Ma}(\mathrm{MSWD}=0.26$, Fig. $7 \mathrm{~d}$; Table 5 LOM), whilst three analyses from the cores yield a concordia age of $426 \pm 25 \mathrm{Ma}(\mathrm{MSWD}=4.4$, Fig. 7d; Table 5 LOM).

(e) Zircon crystals from sample WPP $(\sim 150-250 \mu \mathrm{m}$, aspect ratios 1:2-1:5) are euhedral and normal to long prismatic. Most grains are devoid of inherited cores and have prominent oscillatory zoning. Luminescence is variable, but mostly moderate to weak. Some grains display growth zoning that is progressively less luminescent towards the margins. Irregular, perturbed oscillatory zoning in some grains may be ascribed to sub-solidus recrystallisation and corrosion (Fig. 11). Thirteen LA-MC-ICP-MS U-Pb measurements on nine crystals were made (Table 5 WPP). Eleven analyses from the oscillatory-zoned zircon yield a concordia age of $345 \pm 4 \mathrm{Ma}$ (MSWD = 2.0, Fig. 12; Table 5 WPP). A few grains contain substantially older cores. Two analyses from these cores yield a concordia age of $462 \pm 18$ and $533 \pm 19$ Ma (Fig. 12; Table 5 WPP), which are interpreted as the age of inherited xenocry sts.

Both the crystallisation concordia age of c. $345 \mathrm{Ma}$ and the ages of inherited cores agree with previously published zircon U-Pb ages from this magmatic cycle (Gawęda 2008; Burda and Klötzli 2011; Burda et al. 2013a).

\section{Discussion}

\section{Recycling of the magmatic material}

The presence of sharply bounded biotite monzogranite enclaves (Fig. 2d) and cumulate enclaves inside the younger granites (predominantly in the High Tatra Mountains; Gawęda 2008, 2009), together with the magmatic brecciation (Fig. 3c, d) and small syn-magmatic faults (Gawęda and Szopa 2011), is used as criteria for the activity of density currents, initiated by magma flow and the redistribution of formerly crystallised material during flow sorting (Solgadi and Sawyer 2008).

That, together with two-step crystallisation of matrix $\mathrm{K}$-feldspars, both showing inverted $\mathrm{Ba}$ zonation, with preserved mineral resorption features (Fig. 3a, b, e, f), are 

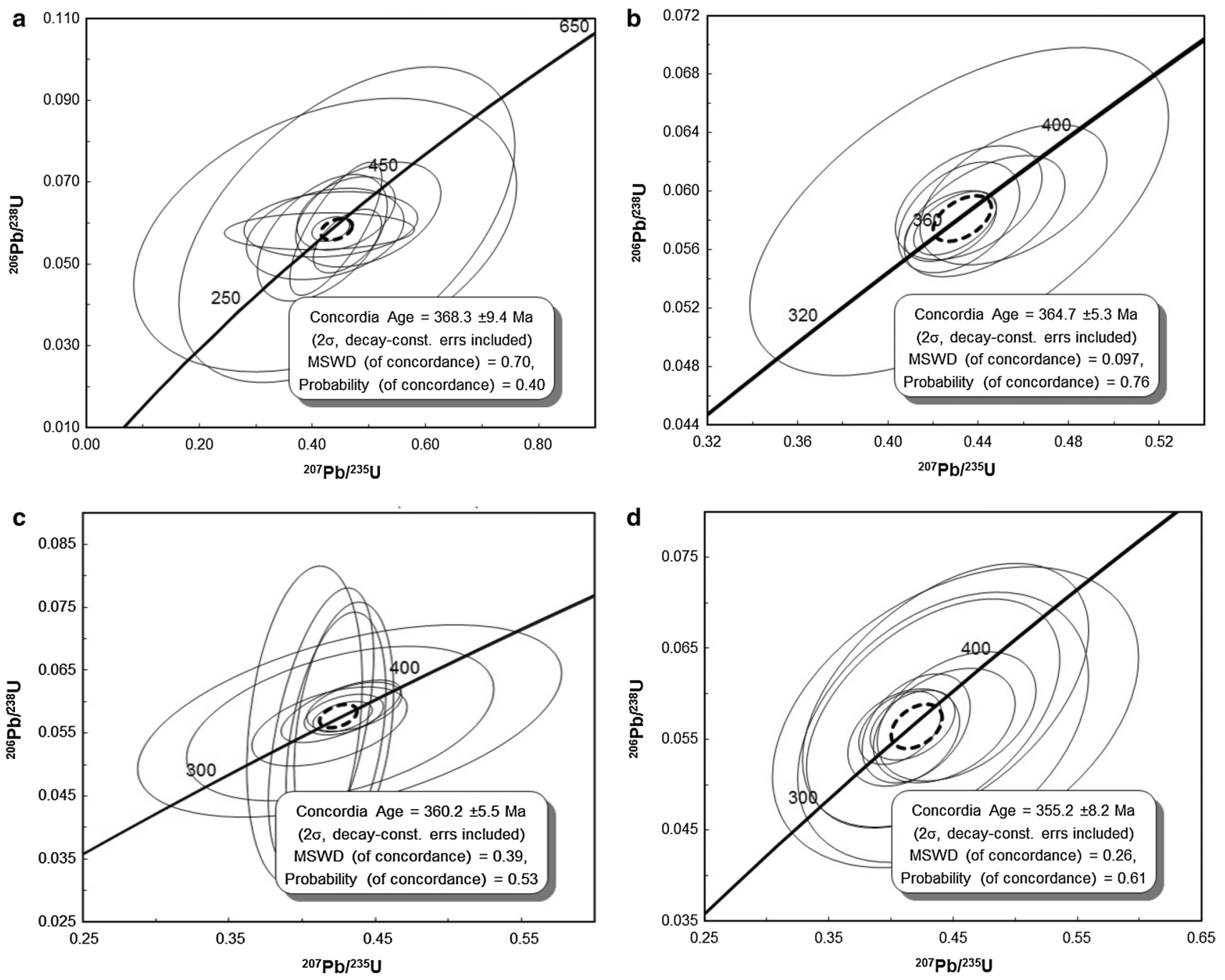

Fig. 7 Concordia plots of LA-MC-ICP-MS zircon analytical results of four samples of the Tatra granites: KOS (Koszysta granitoid, High Tatra Mountains), WG (Wołowiec Mt., Western Tatra Mountains),

evidence of continuous magma influx and crystallisation. The published zircon data also point to the recycling of magmatic material, presented both in CL images and in $\mathrm{U}_{-}$ $\mathrm{Pb}$ geochronology: the common presence of $361 \mathrm{Ma}$ cores rimmed by 345 overgrowths (Gawęda 2008) and $350 \mathrm{Ma}$ cores rimmed by 337 Ma mantles (Burda et al. 2013a) was noted in the High Tatra granitoids.

As the incremental growth of granitoid plutons often involves the remobilisation of older magmatic components, subsequently emplaced (e.g. Oberc-Dziedzic et al. 2013), we can suppose that the following magma fractions are crystal mushes of high viscosity containing the recycled earlier formed components (the "petrological cannibalism" of Cashman and Blundy 2013) as well as country-rock xenoliths, locally deforming the magmatic fabric (Gawęda and Szopa 2011).
RP (Rohacz Płaczliwy Mt., Western Tatra Mountains) and LOM (Łomnica Mt., High Tatra Mountains)

\section{Problem of source rocks to the granitoid magma}

Major elements compositions can point out to the nature of the melted material (Patiño Douce 1999). Melts derived from amphibolites and mafic pelites have lower alkali and aluminium contents, but are enriched in calcium, titanium, iron and magnesium. The analysed granitoids plot in the field of melts generated from an amphibolite-pelite source (calc-alkali I-type granites; Patiño Douce 1999; Fig. 13). These rocks are, however, peraluminous, and are typical magmatic products of a partially molten felsic source.

In primitive mantle-normalised plots (Sun and McDonough 1989) negative $\mathrm{Nb}$ and $\mathrm{Ta}$ anomalies are observed (Fig. 14), suggesting a typical arc setting (Thirwall et al. 1994), consistent with their plotting in VAG field on the Pearce et al. (1984) diagram (Fig. 15). 


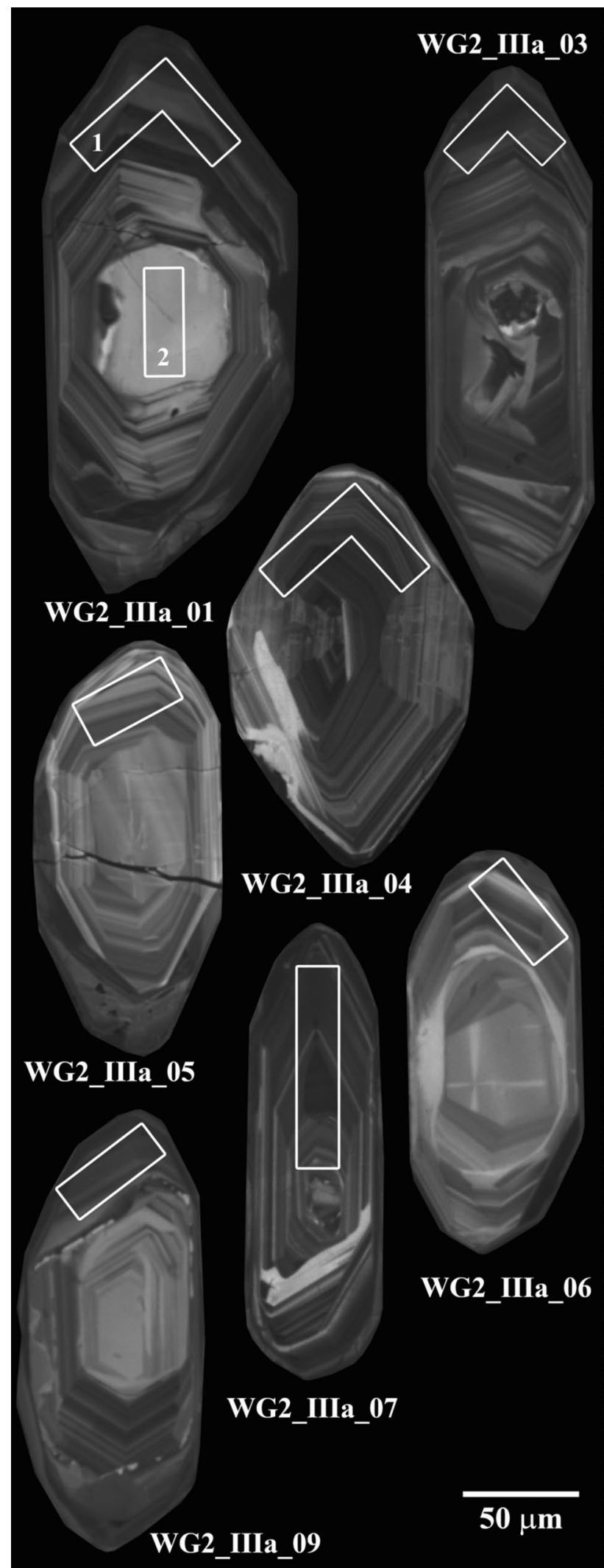

Fig. 8 Cathodoluminescence (CL) images of zircon crystals from WG sample (Wołowiec Mt., Western Tatra Mountains)

\section{Geochronological framework}

Magmatic crystallisation ages plot in the wide time span of $368 \pm 9 \mathrm{Ma}$ (for the Koszysta granite-KOS; Fig. 7a) to $345 \pm 4 \mathrm{Ma}$ (for the High Tatra graniteWPP; Fig. 12). The oldest age is consistent with the age of hybrid magmatic precursors from the Western Tatra Mountains (368 $\pm 8 \mathrm{Ma}$; Burda et al. 2011) and the age of the so-called Goryczkowa granite $(371 \pm 6 \mathrm{Ma}$; Burda et al. 2013b) which, together with geochemical evidence (Fig. 4a-c), suggest a genetic link between these granitoid rocks. The intermediate ages $(364 \pm 5,360 \pm 5$ and $355 \pm 8 \mathrm{Ma}$ ) cover the same time span as defined for two episodes of partial melting, migmatisation and melt expulsion in the Western Tatra Mountains (c. 365$358 \mathrm{Ma}$; Burda and Gaweda 2009). On the Batchelor and Bowden (1985) genetic diagram hybrid diorites (mafic precursors) and granitoids showing ages older than $355 \mathrm{Ma}$ (classified as common Tatra type) plot together as a pre-plate collisional suite, overlapping partly the postcollisional granites field (Fig. 16). It is possible that the older interval of granitoid magmatism represents the preplate collision to collision stage with a Proto-Carpathian Terrane (Gawęda and Golonka 2011), started from mafic precursors intrusion at c. $370 \mathrm{Ma}$ and developed to voluminous granitoid magmatism associated with the partial melting of the metamorphic host rocks. In geodynamic terms in the time span c. 370-355 Ma the subductionrelated diorite-granitoid magmatism had been developed, associated with subduction and partial melting of the accretionary prism.

The youngest granitoids presented here show a magmatic concordia age of $345 \pm 4 \mathrm{Ma}$ (Figs. 11, 12), consistent with former $\mathrm{U}-\mathrm{Pb}$ zircon dating $(345 \pm 5 \mathrm{Ma}$ in Gawęda 2008; $350 \pm 5,345 \pm 6,337 \pm 6$ Ma in Burda et al. 2013a, b). Taking into account the field relationships and textures, the youngest granitoids (c. 350-340 Ma) probably developed as a set of discrete magma pulses in the regional-scale shear zone following one another (Fig. 17ac). Such a scenario is imprinted in the magmatic textures, showing a recycling of magmatic material (Figs. 2d, 3a, e, g). On the Batchelor and Bowden (1985) diagram these rocks plot as post-collisional, late orogenic magmas (Fig. 16), suggesting formation during the final stages of granitoid magmatism.

\section{Thermal consequences for the granitoid intrusion model}

The general tongue-shaped geometry of the granite intrusion and the concordance of magmatic and metamorphic foliations throughout the granitoid body (Kohut 


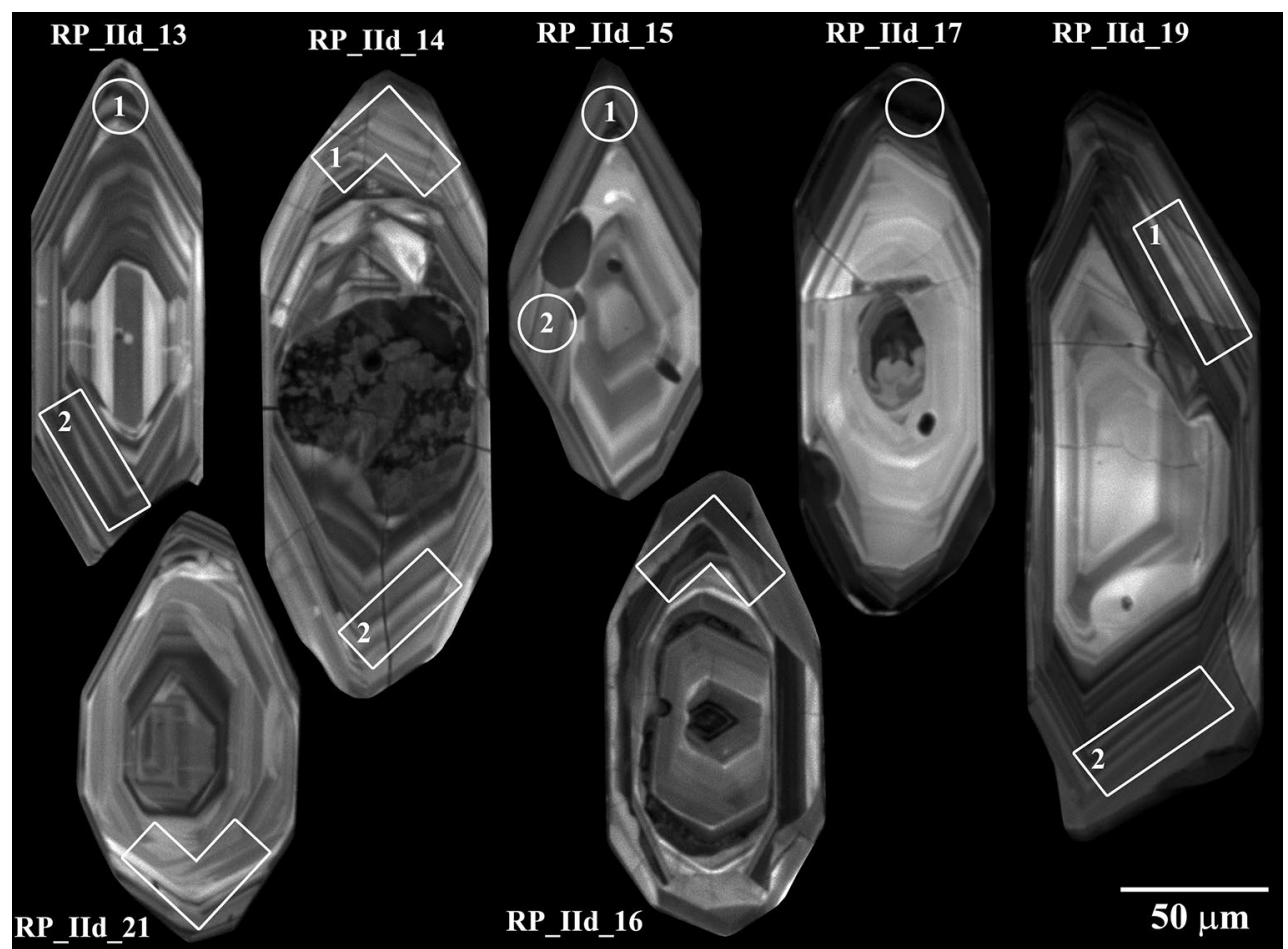

Fig. 9 Cathodoluminescence (CL) images of zircon crystals from RP sample (Rohacz Płaczliwy Mt., Western Tatra Mountains)

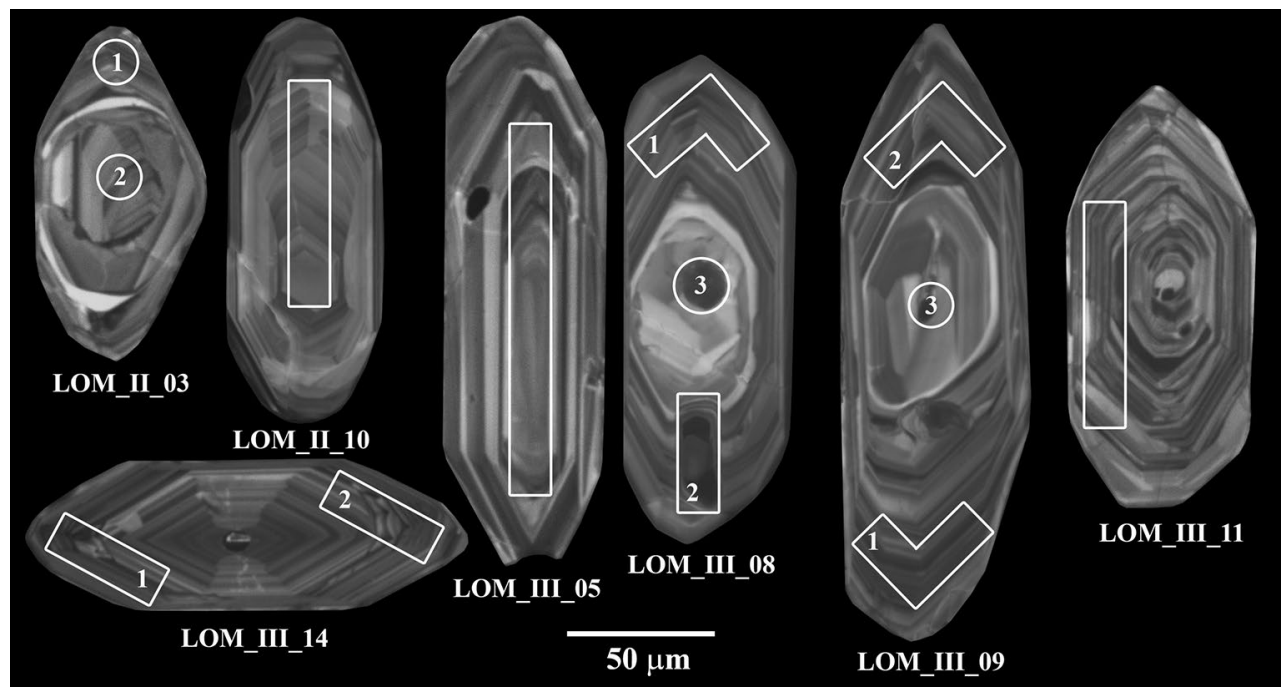

Fig. 10 Cathodoluminescence (CL) images of zircon crystals from LOM sample (Łomnica Mt., High Tatra Mountains)

and Janak 1994; Gawęda and Szopa 2011) suggest that the whole Tatra granitoid intrusion could be a result of repeated injections of small magma batches. The complex post-Variscan history of the Tatra Mountains precludes a determination of the internal zonation of the Tatra granitoid pluton. However, observations from selected vertical sections (Gawęda and Szopa 2011) suggest rather chaotic relationships between successive tabular magma injections (Fig. 17a-c). This is consistent with previously published thermal modelling data for multilayered tabular Variscan intrusions (Diaz Alvarado et al. 2013). All these magma batches show similar zircon saturation temperatures $750-850{ }^{\circ} \mathrm{C}$ (Tables 3,4$)$. The presence of inherited zircon crystals indicates that the melt temperature cannot 


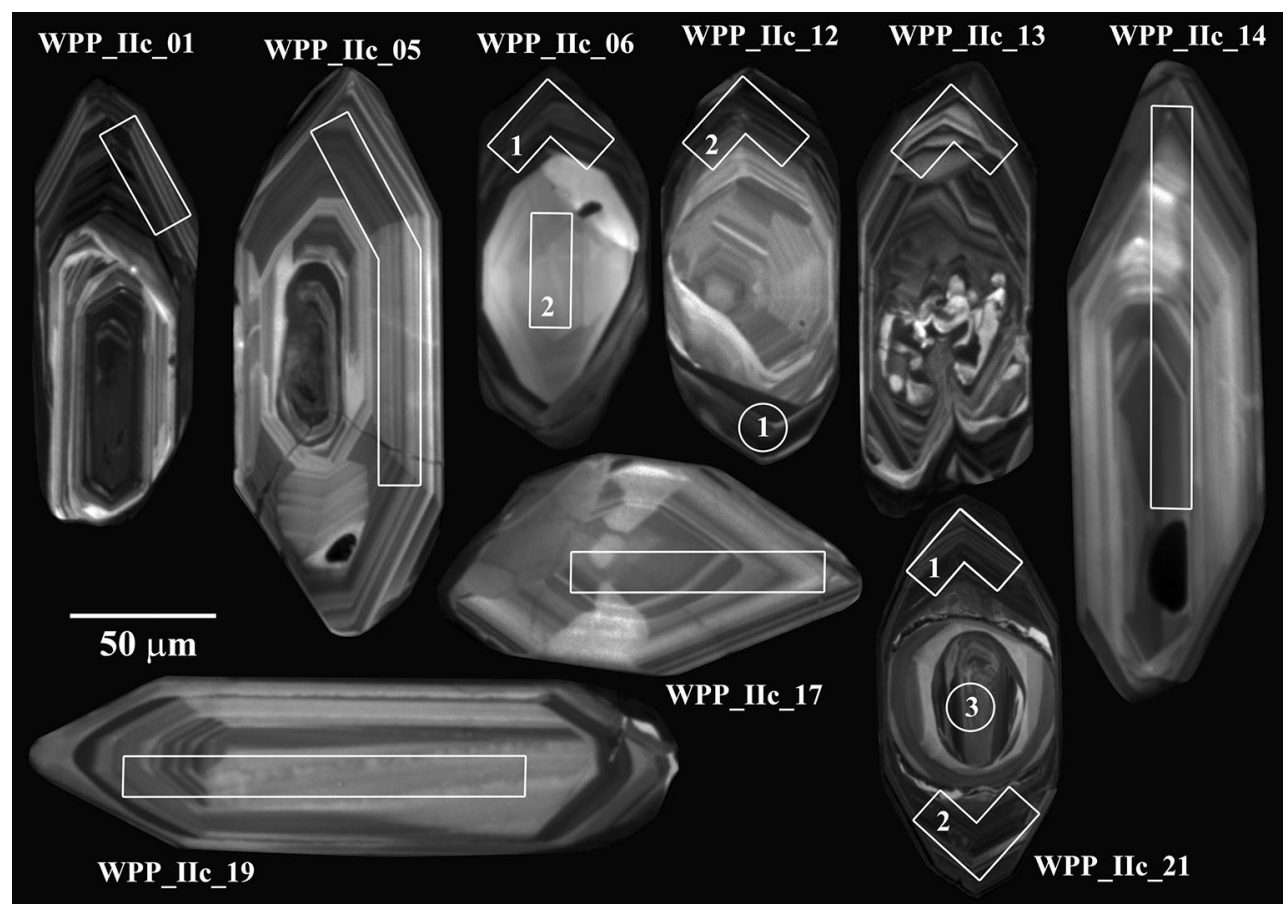

Fig. 11 Cathodoluminescence (CL) images of zircon crystals from WPP sample (Wielki Piarg, High Tatra Mountains)

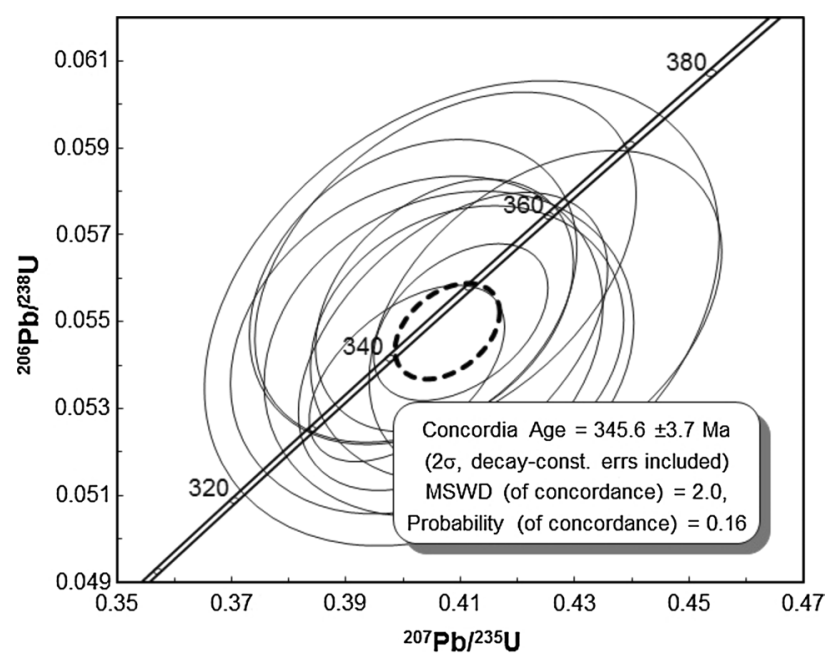

Fig. 12 Concordia plot of LA-MC-ICP-MS zircon analytical results from WPP granitoid sample

be higher than $900{ }^{\circ} \mathrm{C}$ and that zircon resorption was restricted. However, the partial resorption of zircon crystals cannot be wholly excluded, as some zircon should be dissolved during repeated magma injections to keep the zircon saturation level (Diaz Alvarado et al. 2013). The small size and rarity of these inherited zircon population allow to suppose that their presence only slightly influences the calculated $T_{\mathrm{Zr}}$ temperatures; anyway, the calculated temperatures, especially those above $800{ }^{\circ} \mathrm{C}$, may

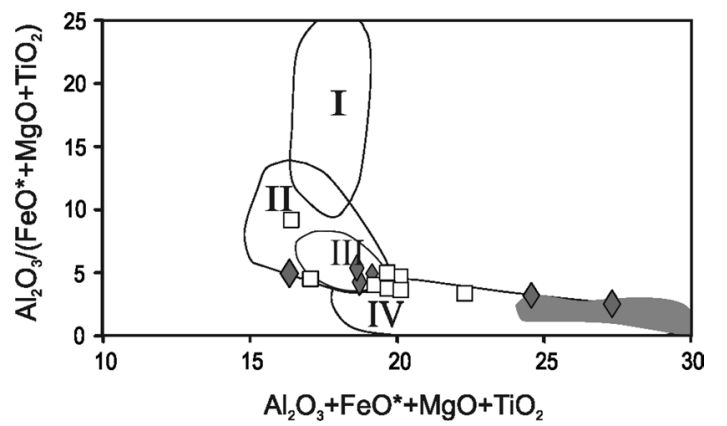

Fig. 13 Chemical composition of Goryczkowa granitoids in major oxide diagrams after Patiño Douce (1999). Outlined fields denote compositional fields of experimental melts derived from partial melting of felsic pelites, metagreywackes and amphibolites. White squares common Tatra granite, grey diamonds High Tatra granites, grey area mafic precursors (according to Gawęda et al. 2005)

be slightly overestimated, and most of the granite batches in fact belong to the "cold" granites, interleaving with "hot" ones (Miller et al. 2003), with no inherited zircon present (Table 4, analyses WPP-3 and WPP-4). Assuming that the granitoid magma started crystallisation during emplacement, the $T_{\mathrm{Zr}}$ data point to the presence of super-solidus conditions for a long time period (approximately $30 \mathrm{Ma}$, representing a difference between the oldest and the youngest $\mathrm{U}-\mathrm{Pb}$ zircon ages). The stable thermal field for such a long time explains the relatively low precision of calibrated ages $( \pm 9 ; \pm 8 \mathrm{Ma})$, especially in 
Fig. 14 Primitive mantle-normalised (Sun and McDonough 1989) multi-element "spider" diagrams for Tatra granitoids and their mafic precursors

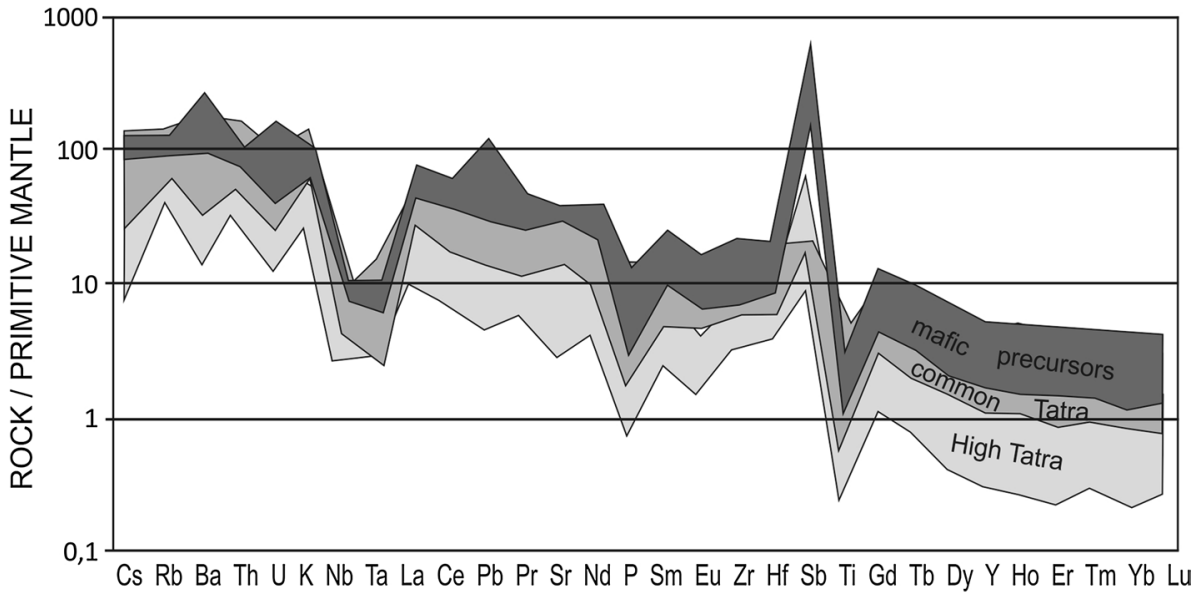

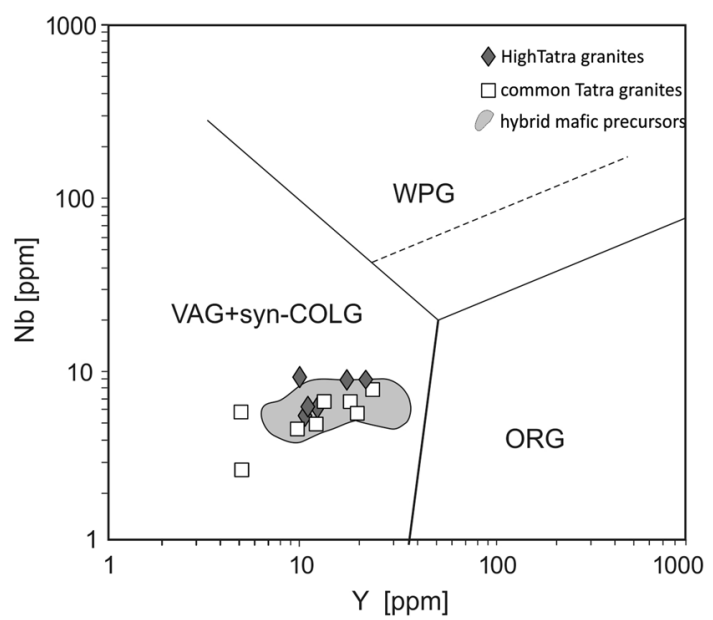

Fig. 15 Plot of the Tatra Mountains granitoids in the Pearce et al. (1984) discrimination diagram. VAG volcanic arc granites, syn-COLG syn-collisional granites, $W P G$ within-plate granites, $O R G$ ocean ridge granites. Symbols as on Fig. 4

the case of the older magmatic components (e.g. KOS sample), which underwent further heating and re-equilibration during continuous magma supply into the active shear zone. In such cases the calculated reproducibility of the zircon ages is outside of the analytical reproducibility due to the maintenance of a long-lasting high-T environment leading to an individual "closure" of the U/ $\mathrm{Pb}$ system in the zircon crystals depending on the crystal chemistry, micro-chemical environment, fluids, fluctuating thermal fields, etc.

\section{Geodynamic scenario}

Closure of the Rheic Ocean, the Variscan orogeny and formation of Pangea supercontinent were initiated by the collision of microcontinents with Laurussia. In the Central

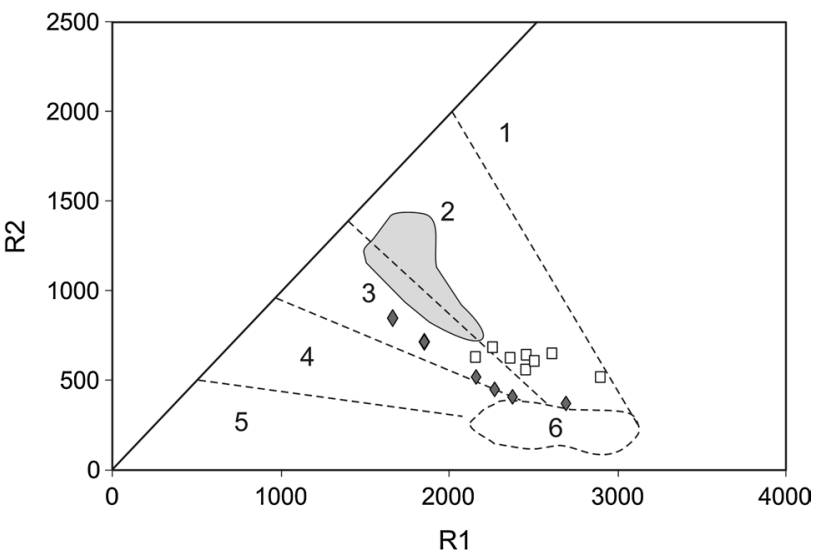

Fig. 16 Multicationic R1-R2 diagram after De La Roche et al. (1980), with fields numbered according to Batchelor and Bowden (1985) showing the position of the Tatra Mountains granitoids. 1 Mantle fractionates, 2 pre-plate collision suites, 3 post-collision suites, 4 late orogenic magmas, 5 anorogenic suites, 6 syncollisional (anatectic) suites. $\mathrm{R} 1=4 \mathrm{Si}-11(\mathrm{Na}+\mathrm{K})-2(\mathrm{Fe}+\mathrm{Ti})$; $\mathrm{R} 2=6 \mathrm{Ca}+2 \mathrm{Mg}+\mathrm{Al}$. Symbols as on Fig. 4

Europe these microcontinents/microplates included TeplaBarandien, Lugia, Brunovistulia and Proto-Carpathia (called also the Tatra composite terrane; Kalvoda and Babek 2010). The Proto-Carpathian Terrane roughly corresponds to the present-day Tatricum unit within the Central Western Carpathians (Ebner et al. 2008), which is an amalgamation of the continental Cadomian crust (Burda and Klötzli 2011), the volcanic arc-related granitoids, formed in the time interval 370-345 Ma and obducted RhenoHercynian fragments of the oceanic crust (amphibolites and eclogites; Janák et al. 1996; Gawęda et al. 2000), representing the arc, originally located above the subduction zone.

Possibly some of the granitic pulses originated in the outer zone of the arc, close to the Rheic oceanic trench, 


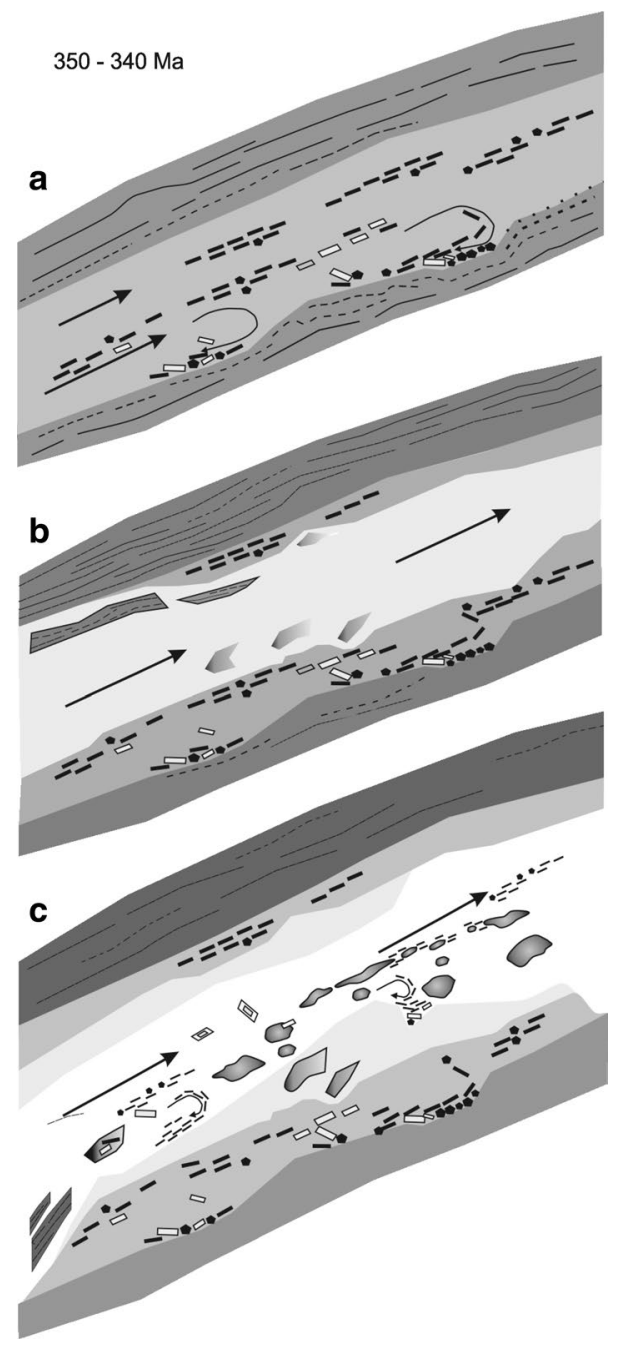

Fig. 17 Schematic illustration of the High Tatra granitoid intrusion development, involving magma mixing, flow segregation and modal layers formation (a), wall-rocks crushing (including granites) and material recycling (b), mafic blobs invasion, magma mixing, flow segregation and cumulate formation (c)

in relatively low-temperature conditions, although high enough to melt the felsic component of the arc (Fig. 18). The temperature increased in the inner (and deeper) part of the supra-subduction zone, what resulting in the melting of more mafic lower crust and formation of dioritic magmas (Gawęda et al. 2005). The supply of the hightemperature dioritic melts promoted further melting and supply to the shear zone of successive pulses of magma, differing in composition and interacting with each other. The interaction of felsic and mafic magmas from the different depths of the same arc resulted in mixing-mingling textures, common in all Tatra granitoids (Burda et al. 2011; Gawęda and Sikorska 2009). Such a scenario allows us to understand the presence of coeval (dated at ca. $368 \mathrm{Ma}$ ) dioritic intrusions with lower crustal/upper mantle characteristics (Gawęda et al. 2005; Burda et al. 2011) and relatively felsic, peraluminous Koszysta granitoids (Table 3) as well as the coexistence of "hot" and "cold" granite batches (Miller et al. 2003). That allows also to resolve the historical debate about the differences between "Koszysta-type" and "Goryczkowa-type" granites (Morozewicz 1914), representing the same magmatic episode, but differing in formation depth of the parent magma.

Continuous subduction and collision of the ProtoCarpathian Terrane with the arc resulted in continuing magma pulses, using the same migration path-the active shear zone. Final closure of the back-arc RhenoHercynian basin and collision with Laurussia were connected with the change of the extensional regime into a compressional one and took place at ca. $340 \mathrm{Ma}$ (Burda et al. 2013a).

The suggested association of the Proto-Carpathian Terrane with the Rheno-Hercynian back-arc basin (Gaweda and Golonka 2011) is supported by the geochemical similarities of amphibolites from the Western Tatra Mountains and Orlica-Śnieżnik Dome in Sudety Mountains (Gawęda et al. 2000; Winchester et al. 2002) and the time of the granitoid magmatism and associated metamorphic events (Burda et al. 2013b). The Tatra Mountains and the whole Tatricum unit seem to be the eastern prolongation of the Armorican Terrane Assemblage (ATA; Tait et al. 1997; Kalvoda and Babek 2010).

The inherited zircon cores provide information on the origin of the melted rocks. Among the analysed inherited cores the 533 and $518 \mathrm{Ma}$ ages are a result of the
Fig. 18 Geodynamic scenario for the development of the backarc Rheno-Hercynian basin and subduction of the oceanic plate under an arc with associated melting of the lower and upper crust and the melt migration along the shear zone

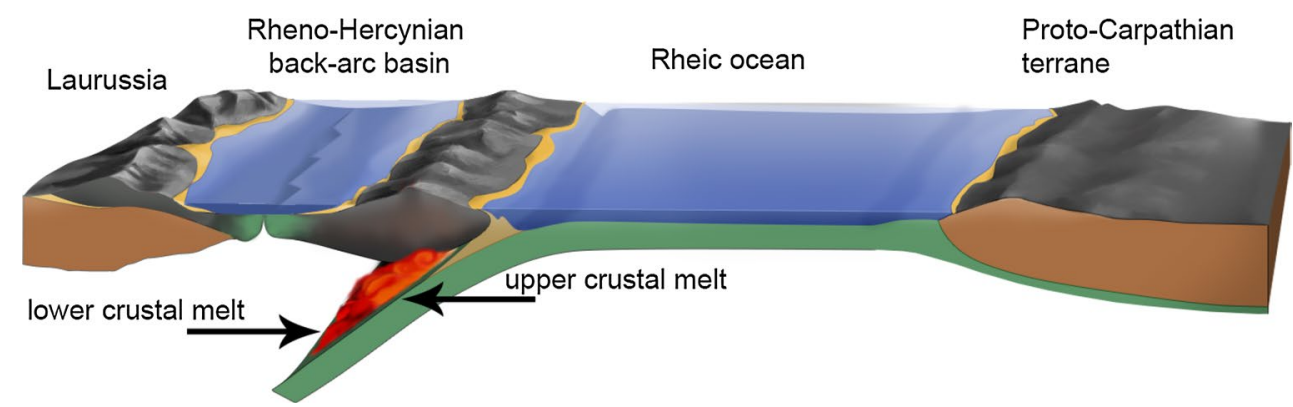


recycling of Cadomian granitoid rocks (Burda and Klötzli 2011) and/or metasedimentary rocks (Kohut et al. 2008). The collision of the promontory of Gondwana with Cadomian consolidation with small terranes marked the onset of the Variscan collision in Central Europe (Kroner and Romer 2013). The presence of magmatic inherited zircon cores with ages at 462 and 426 Ma coincides with previously noted inherited ages of 460, $450 \mathrm{Ma}$ (Burda et al. 2013a), 433 and $412 \mathrm{Ma}$ (Burda et al. 2013b) and WR Rb-Sr age of the gneiss from Goryczkowa (Burchart 1970) and many other localities in Tatric and Veporic units (Burda et al. 2013b and references therein). That suggests the melting of rocks of Avalonian affinity from the very beginning of the granitoid magmatism and supports the thesis that some portions of the Tatricum unit had to belong to the ribbon-like microplate rifted from the southern margin of Laurussia or an active character of that margin.

\section{Conclusions}

1. The Tatra Mountains granitoid body is a composite pluton, formed by repeated magma influx into an active shear zone over a relatively long geological time span (c. $30 \mathrm{Ma}$ ). As the temperature of magma batches was quite constant $\left(\sim 800{ }^{\circ} \mathrm{C}\right)$, the super-solidus conditions were kept for a long time period and allowed the partial resorption and petrological recycling of the former granitoid batches during the following magma injections.

2. The long-lasting granitoid magmatism was a result of multi-step subduction of oceanic crust and collision of Proto-Carpathian Terrane with a volcanic arc and finally with Laurussia. The differences in granitoids composition could be interpreted in terms of different depths of crustal melting: more felsic granitic pulses originated in the outer zone of the arc, close to the Rheic oceanic trench, under relatively low-temperature conditions, whilst in the inner (and deeper) part of the supra-subduction zone, more mafic lower crust was melted, giving rise to dioritic magmas.

3. The presence of inherited zircon cores allows us to suggest the recycling the Cadomian crust, metasedimentary rocks of volcanic arc provenance and rocks of Avalonian affinity. The last observation allows us to suppose that at least some parts of the Tatricum unit belonged to a ribbon-like microplate rifted from the southern margin of Laurussia or an active character of the southern Laurussia margin.
Acknowledgments Dr Piotr Dzierżanowski and Mrs Lidia Jeżak are thanked for their help during microprobe work. Ms Natalia Degórska is gratefully acknowledged for the preparation of graphics for geodynamic scenario. Prof Ray MacDonald (Lancaster University, University of Warsaw) is thanked for the English correction and discussion during the manuscript preparation. Comments of the reviewers: M. Kusiak and K. Breiter as well as editorial comments of prof Wolf-Christian Dullo, led to a clearer presentation of the paper and are deeply acknowledged. This study was financially supported by National Science Centre (NCN) Grant 2012/07/B/ST10/04366 (given to $\mathrm{AG})$.

Open Access This article is distributed under the terms of the Creative Commons Attribution 4.0 International License (http://creativecommons.org/licenses/by/4.0/), which permits unrestricted use, distribution, and reproduction in any medium, provided you give appropriate credit to the original author(s) and the source, provide a link to the Creative Commons license, and indicate if changes were made.

\section{References}

Batchelor RA, Bowden P (1985) Petrogenetic interpretation of granitoid rock series using multicationic parameters. Chem Geol 48:43-55

Broska I, Petrik I, Be`eri-Shlevin J, Majka J, Bezak V (2013) Devonian/Mississipian I-type granitoids in the Western Carpathians: a subduction-related hybrid magmatism. Lithos 162-163:27-36. doi:10.1016/j.lithos.2012.12.014

Burchart J (1970) Rocks of the Goryczkowa "crystalline island" in the Tatra Mountains [Skały krystaliczne Wyspy Goryczkowej w Tatrach]. Stud Geol Pol 32:7-183 (in Polish, English abstract)

Burchart J (1972) Fission-track age determination of accessory apatite from the Tatra Mountains, Poland. Earth Planet Sci Lett 15(3):418-422

Burda J, Gawęda A (2009) Shear-influenced partial melting in the Western Tatra metamorphic complex: geochemistry and geochronology. Lithos 110:373-385. doi:10.1016/j.lithos.2009.01.010

Burda J, Klötzli U (2011) Pre-Variscan evolution in the Western Tatra Mountains: new insides from U-Pb zircon dating. Mineral Petrol 102:99-115. doi:10.1007/s00710-011-0176-4

Burda J, Gawęda A, Klötzli U (2011) Magma hybridization in the Western Tatra Mountains granitoid intrusion (S-Poland, Western Carpathians). Mineral Petrol 103:19-36. doi:10.1007/ s00710-011-0150-1

Burda J, Gawęda A, Klötzli U (2013a) U-Pb zircon age of the youngest magmatic activity in the High Tatra granite. Geochronometria 40(2):134-144. doi:10.2478/s13386-013-0106-9

Burda J, Gawęda A, Klötzli U (2013b) Geochronology and petrogenesis of granitoid rocks from the Goryczkowa Unit, Tatra Mountains (Central Western Carpathians). Geol Carpath 64(6):419435. doi:10.2478/geoca-2013-0029

Cashman K, Blundy J (2013) Petrological cannibalism: the chemical and textural consequences of incremental magma body growth. Contrib Mineral Petrol 166:703-729. doi:10.1007/ s00410-013-0895-0

Coleman DS, Gray W, Glazner AF (2004) Rethinking the emplacement and evolution of zoned plutons: geochronologic evidence for incremental assembly of the Tuolumne Intrusive Suite, California. Geology 32:433-436 
De la Roche H, Leterrier J, Grandclaude P, Marchal M (1980) A classification of volcanic and plutonic rocks using R1, R2-diagrams and major element analysis - its relationships with current nomenclature. Chem Geol 29:183-210

Diaz Alvarado J, Fernandez C, Castro A, Moreno-Ventas I (2013) SHRIMP U-Pb geochronology and thermal modelling of multilayer granitoid intrusions: implication for the building and thermal evolution of the Central System batholith, Iberian massif, Spain. Lithos 175-176:104-123

Ebner F, Vozarova A, Kovacs S, Krautner H, Krstic B, Szederkenyi T, Jamicic D, Balen D, Belak M, Trajanova M (2008) DevonianCarboniferous pre-flysch and flysch environments in the Circum Pannonian Region. Geol Carpath 59(2):159-195

Gaab A, Poller U, Todt W, Janák M (2003) Geochemical and isotopic characteristics of the Murán Gneiss Complex, Veporic Unit (Slovakia). J Czech Geol Soc 48(1-2):52

Gawęda A (2008) Apatite-rich enclave in the High Tatra granite, Western Carpathians: petrological and geochronological study. Geol Carpath 59(4):295-306

Gawęda A (2009) Enclaves in the High Tatra Granite. University of Silesia publishing House, Monographic series 2637, Katowice (in Polish, English abstract)

Gawęda A, Golonka J (2011) Variscan plate dynamics in the circumcarpathian area. Geodin Acta 24(3-4):141-155. doi:10.3166/ ga.24.141-145

Gawęda A, Sikorska M (2009) Alkali feldspar megacrysts from the High Tatra granite-indicators of magma mixing/mingling processes. Mineral Spec Pap 35:82

Gawęda A, Szopa K (2011) The origin of magmatic layering in the High Tatra granite, Central Western Carpathians-implications for the formation of granitoid plutons. Earth Env Sci T Royal Soc Edinb 103:129-144. doi:10.1017/S1755691012010146

Gawęda A, Włodyka R (2012) The origin of post-magmatic Ca-Al minerals in granite-diorite mingling zones: the Tatra granitoid intrusion, Western Carpathians. N Jhb Mineral Abh 190(1):2947. doi:10.1127/0077-7757/2012/0228

Gawęda A, Winchester JA, Kozłowski K, Narębski W, Holland JG (2000) Geochemistry and paleotectonic setting of amphibolites from the Western Tatra Mountains, southern Poland. Geol J 35:69-85

Gawęda A, Doniecki T, Burda J, Kohut M (2005) The petrogenesis of quartz-diorites from the Tatra Mountains (Central Western Carpathians): an example of magma hybridisation. N Jhb Mineral Abh 1:95-109. doi:10.1127/0077-7757/2005/0181/0005

Gawęda A, Szopa K, Chew D (2014) LA-ICP-MS U-Pb dating and REE patterns of apatite from the Tatra Mountains, Poland as a monitor of the thermal events. Geochronometria 41:306-314. doi:10.2478/s13386-013-0171-0

Glazner AF, Bartley JM, Coleman DS, Gray W, Taylor RZ (2004) Are plutons assembled over millions of years by amalgamation from small magma chambers? GSA Today 14:4-11. doi:10.1330/1052-5173(2004)014<0004:APAOMO>2.0.CO;2

Golonka J, Gawęda A (2012) Plate tectonic evolution of the southern margin of Laurussia in the Paleozoic. Tecton Recent Adv Ch 10:261-282. doi:10.5772/50009

Golonka J, Krobicki M, Oszczypko N, Ślączka A, Słomka T (2003) Geodynamic evolution and paleogeography of the Polish Carpathians and adjacent areas during Neo-Cimmerian and preceding events (latest Triassic-earliest Cretaceous). In: McCann T, Saintot A (eds) Tracing tectonic deformation using the sedimentary record, vol 208. Geological Society, London, Special Publication, pp 138-158

Grabowski J, Gawęda A (1999) Preliminary paleomagnetic study of the High Tatra granites, Central Western Carpathians, Poland. Geol Quaterly 43(3):263-276
Hibbard MJ (1991) Textural anatomy of twelve magma-mixed granitoid systems. In: Didier J, Barbarin B (eds) Enclaves and granite petrology developments in petrology, vol 13. Elsevier, Amsterdam, pp 431-444

Janák M, O`Brien PJ, Hurai V, Reutel C (1996) Metamorphic evolution and fluid composition of garnet-clinopyroxene amphibolites from the Tatra Mountains, Western Carpathians. Lithos 39:57-79

Janák M, Finger F, Plašienka D, Petrik I, Humer B, Meres S, Luptak B (2002) Variscan high P-T recrystallization of Ordovician granitoids in Veporic Unit (Nizke Tatry Mountains, Western Carpathians): new petrological and geochronological data. Geolines 14:38-39

Jurewicz E (2005) Geodynamic evolution of the Tatra Mts. and the Pieniny Klippen Belt (Western Carpathians): problems and comments. Acta Geol Pol 55:295-338

Kalvoda J, Babek O (2010) The margin of Laurussia in central and southeast Europe and southwest Asia. Gondwana Res 17:526545. doi:10.1016/j.gr.2009.09.012

Kohut M, Janak M (1994) Granitoids of the Tatra Mts., Western Carpathians: field relations and petrogenetic implications. Geol Carpath 45(5):301-311

Kohut M, Sherlock S (2003) Laser microprobe ${ }^{40} \mathrm{Ar}-{ }^{39} \mathrm{Ar}$ analysis of pseudotachylyte and host-rocks from the Tatra Mountains, Slovakia: evidence for late Palaeogene seismic/tectonic activity. Terra Nova 15(6):417-424

Kohut M, Konecny P, Siman P (2006) The first finding of the iron Lahn-Dill mineralization in the Tatric Unit of the Western Carpathians. Mineral Polon Spec Pap 28:112-114

Kohut M, Poller U, Gurk Ch, Todt W (2008) Geochemistry and U-Pb detrital zircon ages of metasedimentary rocks of the Lower Unit, Western Tatra Mountains (Slovakia). Acta Geol Pol 58:371-384

Kroner U, Romer RL (2013) Two plates-many subduction zones: the Variscan orogeny reconsidered. Gondwana Res 24:298-329. doi:10.1016/j.gr.2013.03.001

Liotta D, Caggianelli A, Kruhl JH, Festa V, Prosser G, Langone A (2008) Multiple injections of magmas along a Hercynian midcrustal shear zone (Sila Massif, Calabria, Italy). J Struct Geol 30:1202-1217. doi:10.1016/j.jsg.2008.04.005

Ludwig KR (2003) Isoplot/Ex version $300 \mathrm{~A}$ geochronological toolkit for Microsoft Excel. Berkeley Geochronol Cent Spec Publ 4:1-74

Middlemost EAK (1985) Magmas and magmatic rocks. An introduction to igneous petrology. Longman Group Ltd., London, New York

Miller CF (1985) Are strongly peraluminous magmas derived from pelitic sedimentary sources? J Geol 93:673-689

Miller CF, McDowell SM, Mapes RW (2003) Hot and cold granites? Implications of zircon saturation temperatures and preservation of inheritance. Geology 31:529-532

Morozewicz K (1914) Über die Tatragranite. N Jb Miner Geol Paläont 39:289-345

Moussallam Y, Schneider DA, Janak M, Thoni M, Holm DK (2012) Heterogeneous extrusion and exhumation of deep-crustal Variscan assembly: geochronology of the Western Tatra Mountains, northern Slovakia. Lithos 144-145:88-108

Nance RD, Gutiérrez-Alonso G, Keppie JD, Linnemann U, Murphy JB, Quesada C, Strachan RA, Woodcock NH (2010) Evolution of the Rheic Ocean. Gondwana Res 17:194-222. doi:10.1016/j. gr.2009.08.001

Oberc-Dziedzic T, Kryza R, Pin Ch, Madej S (2013) Sequential granite emplacement: a structural study of the late Variscan Strzelin intrusion, SW Poland. Int J Earth Sci 102:1289-1304. doi:10.007/s00531-013-0863-y

Paterson SR, Okaya D, Memeti V, Economos R, Miller RB (2011) Magma addition and flux calculations of incrementally 
constructed magma chambers in continental margin arcs: combined field, geochronologic and thermal modelling studies. Geosphere 7(6):1439-1468. doi:10.1130/GES00696.1

Patiño Douce A (1999) What do experiments tell us about the relative contributions of crust and mantle to the origin of granitic magmas? In: Castro A, Fernandez C, Vigneresse JL (eds) Understanding granites. Integrating new and classical techniques, vol 158. Geological Society, London, Special Publication, pp 55-75

Pawley MJ, Collins WJ, Van Kranendonk MJ (2002) Origin of finescale sheeted granites by incremental injection of magma into active shear zones: example from Pilbara Craton, NW Australia. Lithos 61:127-139

Pearce JA, Harris NBW, Tindle AG (1984) Trace elements discrimination diagram for the tectonic interpretation of granitic rocks. J Petrol 25(4):956-983

Pecerillo A, Taylor SR (1976) Geochemistry of Eocene calc-alkaline volcanic rocks from the Kastamonu area, northern Turkey. Contrib Mineral Petrol 58:63-81

Petford N, Cruden AR, Mccaffrey KJW, Vigneresse J-L (2000) Granite magma formation, transport and emplacement in the Earth's crust. Nature 408:669-673. doi:10.1038/35047000

Poller U, Janák M, Kohút M, Todt W (2000) Early Variscan magmatism in the Western Carpathians: U-Pb zircon data from granitoids and orthogneisses of the Tatra Mountains (Slovakia). Int J Earth Sci 89:336-349

Putiš M, Sergeev S, Ondrejka M, Larionov A, Siman P, Spisiak J, Uher P, Paderin I (2008) Cambrian-Ordovician metaigneous rocks associated with Cadomian fragments in the West-Carpathian basement dated by SHRIMP on zircons: a record from the Gondwana active margin setting. Geol Carpath 59(1):3-18

Putiš M, Ivan P, Kohút M, Spišiak J, Siman P, Radvanec M, Uher P, Sergeev S, Larionov A, Méres Š, Rastislav Demko R, Ondrejka M (2009) Meta-igneous rocks of the West-Carpathian basement, Slovakia: indicators of Early Paleozoic extension and shortening events. Bull Soc Géol Fr 180(6):461-471

Ridolfi F, Renzulli A, Puerini M (2010) Stability and chemical equilibrium of amphibole in calc-alkaline magmas: an overview, new thermometric formulations and application to subduction-related volcanoes. Contrib Mineral Petrol 160:45-66. doi:10.1007/ s00410-009-0465-7

Schmidt MW (1992) Amphibole equilibria in tonalite as a function of pressure: an experimental calibration of the Al-in-hornblende geobarometer. Contrib Mineral Petrol 110:304-310
Słaby E, Martin H (2008) Mafic and felsic magma interaction in granites: the Hercynian Karkonosze pluton (Sudetes, Bohemian Massif). J Petrol 49(2):353-391. doi:10.1093/petrology/egm085

Sláma J, Košler J, Schaltegger U, Tubrett M, Gutjahr M (2006) New natural zircon standard for laser ablation ICP-MS U-Pb geochronology. In: Winter Conference on Plasma Spectrochemistry, Tucson Abstract WP05, pp 187-188

Solgadi F, Sawyer EW (2008) Formation of igneous layering in granodiorite by gravity flow: a field, microstructure and geochemical study of the Tuolumne Intrusive Suite at Sawmill Canyon, California. J Petrol 49:2009-2042. doi:10.1093/petrology/cgn056

Stacey JS, Kramers JD (1975) Approximation of terrestrial lead isotope evolution by a two stage model. Earth Planet Sci Lett 26:207-221

Sun SS, McDonough WF (1989) Chemical and isotopical systematics of oceanic basalts: implications for mantle composition and processes. Magmatism in the Oceanic Basins. Geol Soc Lond Spec Pub 42:313-345

Szopa K, Gawęda A, Müller A, Sikorska M (2013) The petrogenesis of granitoid rocks unusually rich in apatite in the Western Tatra Mts. (S-Poland, Western Carpathians). Mineral Petrol 107:609627. doi:10.1007/s00710-012-0262-2

Tait JA, Bachtadse V, Franke W, Soffel HC (1997) Geodynamic evolution of the European Variscan fold belt: paleomagnetic and geological constraints. Geol Rundsch 86:585-598

Thirwall MF, Smith TE, Graham AM, Theodorou N, Hollings P, Davidson JP, Arculus RJ (1994) High field strength element anomalies in arc lava: Source or process? J Petrol 35:819-838

Watson TM, Harrison EB (1983) Zircon saturation revisited: temperature and composition effects in a variety of crustal magma types. Earth Planet Sci Lett 64:295-304

Wiedenbeck M, Alle P, Corfu F, Griffin WL, Meier M, Oberli F, Von Quadt A, Roddick JC, Spiegel W (1995) Three natural zircon standards for U-Th-Pb, Lu-Hf, trace element and REE analyses. Geostand Newsl 19:1-23

Winchester JA, The PACE TMR Network Team (contract ERBFMRXCT97-0136) (2002) Paleozoic amalgamation of Central Europe: new results from recent geological and geophysical investigations. Tectonophysics 360:5-21 